\begin{tabular}{|c|c|c|c|c|}
\hline \multirow{3}{*}{$\begin{array}{l}\text { 2. ECN Category } \\
\text { (mark one) } \\
\text { Supplemental } \\
\text { Direct Revision } \\
\text { [X] Change ECN } \\
\text { Champorary } \\
\text { Temandby } \\
\text { Supersedure } \\
\text { Cancel/Noid }\end{array}$} & \multirow{3}{*}[\begin{array}{l}{[]}\\
{[]}\\
{[]}\\
{[]}\end{array}]{} & $\begin{array}{l}\text { 3. Originator's Name, Organization, MSIN, } \\
\text { and Telephone No. } \\
\text { JE Ellis/Elec Systems/K5- } \\
17 / 375-3627\end{array}$ & $\begin{array}{l}\text { 4. USQ Required? } \\
\text { [] Yes [ No }\end{array}$ & $\begin{array}{l}\text { 5. Date } \\
\text { March 7. } 1997\end{array}$ \\
\hline & & $\begin{array}{l}\text { 6. Project Title/No./Work order No. } \\
\text { LDUA ETN-96-0007 }\end{array}$ & $\begin{array}{c}\text { 7. Bldg./Sys./Fac. No. } \\
\text { N/A }\end{array}$ & 8. Approval Designator \\
\hline & & $\begin{array}{l}\text { 9. Document Numbers Changed by this ECN } \\
\text { (includes sheet no. and rev.) } \\
\text { HNF HAHE-SD-TD-OMM-001 Rev. D }\end{array}$ & $\begin{array}{l}\text { 10. Related ECN No(s). } \\
\text { N/A }\end{array}$ & $\begin{array}{l}\text { 11. Related PO No. } \\
\text { N/A }\end{array}$ \\
\hline
\end{tabular}

\begin{tabular}{|l|l|l|l|l}
\hline 12a. Modification Work & 12b. Work Package & 12c. Modification Work Complete & 12d. Restored to Original Condi-
\end{tabular}

\begin{tabular}{l|l|l|l} 
[] yes fill out & No. & Nion (Temp. or Standby ECN only)
\end{tabular}

\begin{tabular}{c|c|c|c} 
[] Yes (fill out Blk. & N2b) & N/A & N/A
\end{tabular}

$[X]$ No (NA Blks. 12b, $12 c, 12 d)$

13a. Description of Change

Replace all pages with the attached document.

Design Authority/Cog. Engineer Signature \& Date

Design Authority/Cog. Engineer Signature \& Date

13b. Design Basel ine Document? [] Yes $[X]$ No

14a. Justification (mark one)

Criteria Change [] Design Improvement $[X]$ Environmental

As-Found

[]

Facilitate Const

[]

Const. Error/Omission

[] Facility Deactivation

[] Design Error/Omission

[]

14b. Justification Details

Revised the software manual to be consistent with the latest software release.

15. Distribution (include name, MSIN, and no. of copies)

GA Barnes

JE Ellis

LDUA System 4200 File

B $4-51 \quad+5-091$ copy

ga $\mathrm{K} 5-171$ copy

Central Files

K5-22 5 copies

B -07 (Orig. +1 ) 


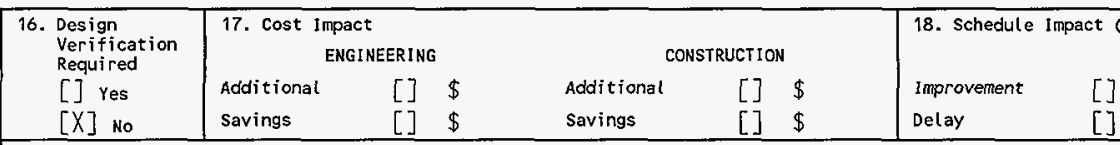

19. Change Impact Review: Indicate the related documents (other than the engineering documents identified on Side 1 ) that will be affected by the change described in Block 13 . Enter the affected document number in Block 20 . SDD/DD

Functional Design Criteria

Operating Specification

Criticality Specification

Conceptual Design Report

Equipment Spec.

Const. Spec.

Procurement Spec.

Vendor Information

OM Manual

FSAR/SAR

Safety Equipment List

Radiation Work Permit

Environmental Impact Statement

Environmental Report

Environmental Permit
[] Seismic/Stress Analysis

[]

[]

[]

[]

[]

[]

[]

[]

[]

[]

[]

[]

[]

[]

[]

Stress/Design Report

Interface Control Drawing

Calibration Procedure

Installation Procedure

Maintenance Procedure

Engineering Procedure

Operating Instruction

Operating Procedure

Operational Safety Requirement

IEFD Drawing

Cell Arrangement Drawing

Essential Material Specification

Fac. Proc, Samp. Schedule

Inspection Plan

Inventory Adjustment Request

[]
[]
[]
[]
[]
[]
[]
[]
[]
[]
[]
[]
[]
[]
[]
[]

Tank Calibration Manual

Health Physics Procedure

Spares Multiple Unit Listing

Test Procedures/Specification

Component Index

ASME Coded Item

Human Factor Consideration

Computer Software

Electric Circuit Schedule

ICRS Procedure

Process Control Manual/Plan

Process Flow Chart

Purchase Requisition

Tickler File

20. Other Affected Documents: (NOTE: Documents (isted below will not be revised by this ECN.) Signatures below indicate that the signing organization has been notified of other affected documents listed below. Document Number/Revision

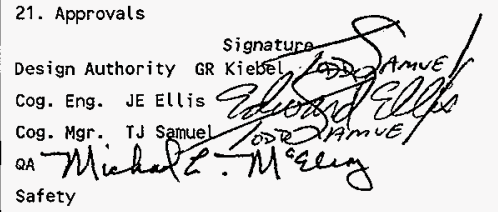

Environ.

other ToRawery

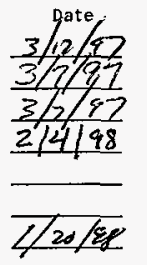

Design Agent

Signature

Date

PE

QA

Safety

Design

Environ.

other

\section{DEPARIMENT OF ENERGY}

signature or a Control Number that

tracks the Approval signature

\section{ADDITIONAL}


OPERATIONS AND MAINTENANCE MANUAL FOR THE LDUA SUPERVISORY CONTROL AND DATA ACQUISITION SYSTEM (LDUA SYSTEM 4200) AND CONTROL NETWORK (LDUA SYSTEM 4400)

\section{GA Barnes}

SGN Eurisys Services Corporation, Richland, WA 99352

U.S. Department of Energy Contract DE-AC06-96RL13200

EDT/ECN: $\quad 617775$

Org Code: 08E00

B\&R Code: EW3120074
UC: 2070

Charge Code: LMB020

Total Pages: 83

Key Words: LDUA, Operations and Maintenance manual(OMM). Data Acquisition and Control System, equipment

Abstract: This document defines the requirements applicable to the operation. maintenance and storage of the Supervisory Control and Data Acquisition System (SCADAS) and Control Network in support of the Light Duty Utility Arm (LDUA) operations.

TRADEMARK DISCLAIMER. Reference herein to any specific commercial product, process, or service by trade name, trademark, manufacturer, or otherwise, does not necessarily constitute or imply its endorsement, recommendation. or favoring by the United States Government or any agency thereof or its contractors or subcontractors.

Printed in the United States of America. To obtain copies of this document, contact: Document Control Services. P.0. Box 950. Mailstop H6-08, Richland WA 99352, Phone (509) 372-2420:

Fax (509) 376-4989.
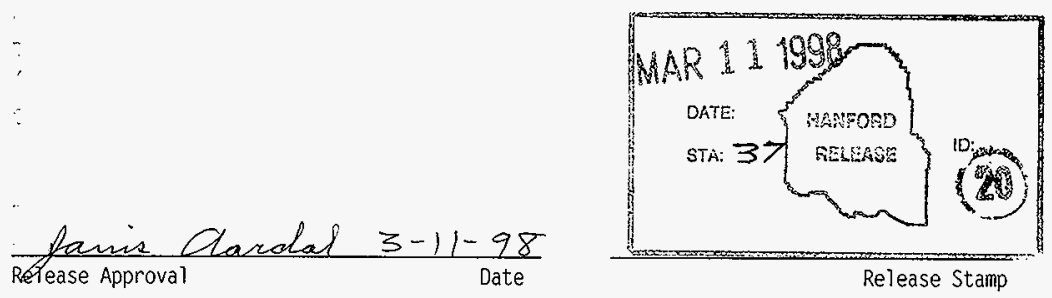

\section{Approved for Public Release}




\section{RECORD OF REVISION}

(1) Document Number

HNF-SD-TD-OMM001 , Rev. 1

(2) Title

Operation and Maintenance Manual for the LDUA Supervisory Control and Data Acquisition System (LDUA System 4200) and Control Network (LDUA System 4400)

CHANGE CONTROL RECORD

(3)

Revision

0.

1 RS
(4) Description of Change - Replace. Add, and Delete EDT-620927, 1/27998

(7) COMPLETE RE-WRITE ECN-677775
Authorized for Release

\begin{tabular}{c|c} 
(5) Cog. & (6) Cog. Mgr. Date \\
Engr.
\end{tabular}

GA Barnes TJ Samuel 3-11-97
S.A. Bainad J.A. Samual (A. Aardal, per 3-11 telecon) 
HNF-SD-TD-OMM-001, Rev. 1

\section{OPERATION AND MAINTENANCE MANUAL FOR THE LDUA SUPERVISORY CONTROL AND DATA ACQUISITION SYSTEM (LDUA SYSTEM 4200) AND CONTROL NETWORK (LDUA SYSTEM 4400)}

$\operatorname{Rev} 1$

J. E. Ellis

April 25, 1997 
HNF-SD-TD-OMM-001, Rev. 1

Page ii

This page is intentionally left blank. 
OPERATION AND MAINTENANCE MANUAL FOR THE LDUA SUPERVISORY CONTROL AND DATA ACQUISITION SYSTEM (LDUA SYSTEM 4200)

AND CONTROL NETWORK (LDUA SYSTEM 4400)

TABLE OF CONTENTS

Page Number

LIST OF FIGURES $\ldots \ldots \ldots \ldots \ldots \ldots \ldots \ldots \ldots \ldots \ldots \ldots \ldots \ldots \ldots \ldots \ldots$ vii

LIST OF FILE LISTINGS $\ldots \ldots \ldots \ldots \ldots \ldots \ldots \ldots \ldots \ldots \ldots \ldots \ldots \ldots \ldots \ldots \ldots \ldots$ vii

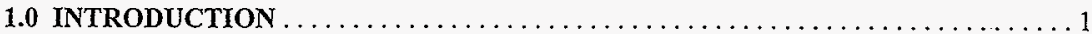

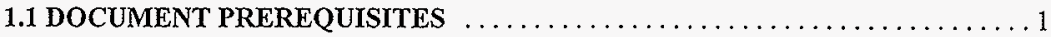

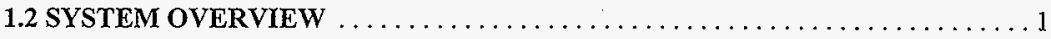

1.2.1 SUPERVISORY CONTROL SYSTEM $\ldots \ldots \ldots \ldots \ldots \ldots \ldots \ldots \ldots \ldots$

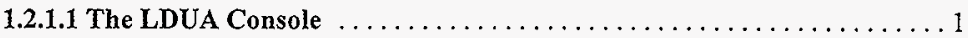

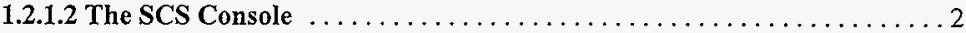

1.2.1.2.1 Commercial robotics simulation software $\ldots \ldots \ldots \ldots \ldots \ldots 2$

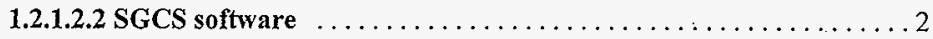

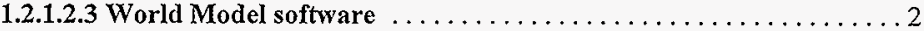

1.2.2 SUPERVISORY DATA ACQUISITION SYSTEM $\ldots \ldots \ldots \ldots \ldots \ldots \ldots 2$

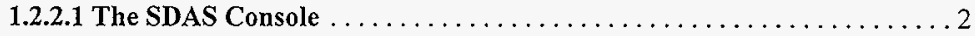

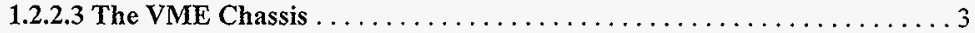

1.2.2.4 Commercial Data Acquisition Software $\ldots \ldots \ldots \ldots \ldots \ldots \ldots \ldots$

1.2.2.5 Commercial real time operating software $\ldots \ldots \ldots \ldots \ldots \ldots \ldots \ldots$

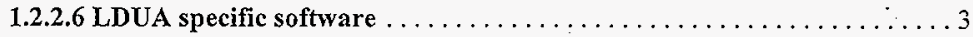

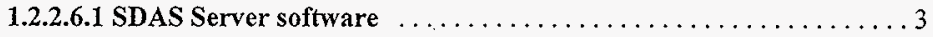

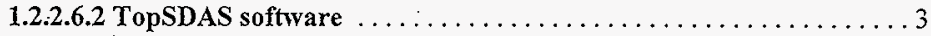

1.2.2.6.3 GuestSDAS software .........................

1.2.3 VIDEO DISPLAY AND RECORDING SYSTEM $\ldots \ldots \ldots \ldots \ldots \ldots \ldots$

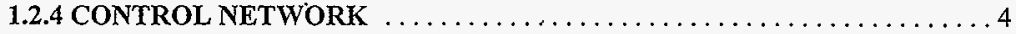

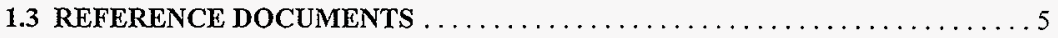

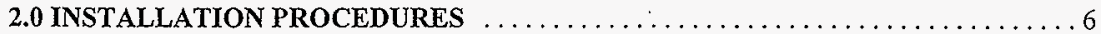

2.1 INSTALLATION OF SCS SOFTWARE $\ldots \ldots \ldots \ldots \ldots \ldots \ldots \ldots \ldots \ldots \ldots \ldots$

2.1.1 INSTALLATION OF LDUA CONSOLE SOFTWARE $\ldots \ldots \ldots \ldots \ldots \ldots$

2.1.2 INSTALLATION OF SCS CONSOLE SOFTWARE $\ldots \ldots \ldots \ldots \ldots \ldots 7$

2.2 INSTALLATION OF SDAS SOFTWARE $\ldots \ldots \ldots \ldots \ldots \ldots \ldots \ldots \ldots \ldots$

2.2.1 INSTALLATION OF SDAS CONSOLE SOFTWARE $\ldots \ldots \ldots \ldots \ldots \ldots 8$

2.2.1.1 CREATE VXWORKS ACCOUNT $\ldots \ldots \ldots \ldots \ldots \ldots \ldots \ldots \ldots$

2.2.1.2 CREATE OPER ACCOUNT $\ldots \ldots \ldots \ldots \ldots \ldots \ldots \ldots \ldots \ldots \ldots$

2.2.1.3 INSTALL SDAS SOFTWARE $\ldots \ldots \ldots \ldots \ldots \ldots \ldots \ldots \ldots \ldots \ldots$

2.2.1.4 INSTALL TIME SERVER SOFTWARE $\ldots \ldots \ldots \ldots \ldots \ldots \ldots \ldots 10$ 
2.2.2 INSTALLATION OF GUEST CONSOLE SOFTWARE $\ldots \ldots \ldots \ldots \ldots 10$ 2.3 CONFIGURATION OF SDAS VME HARDWARE $\ldots \ldots \ldots \ldots \ldots \ldots \ldots \ldots \ldots \ldots$

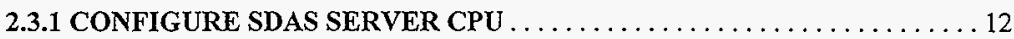

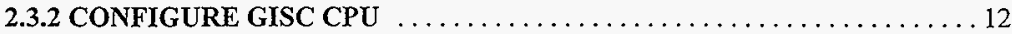

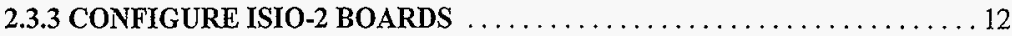

2.3.4 CONFIGURE IP CARRIER BOARDS $\ldots \ldots \ldots \ldots \ldots \ldots \ldots \ldots \ldots$

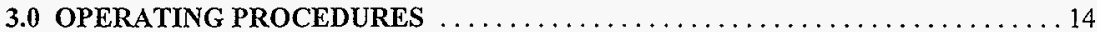

3.1 EQUIPMENT REMOVAL, STORAGE, AND SETUP $\ldots \ldots \ldots \ldots \ldots \ldots \ldots$

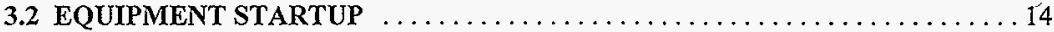

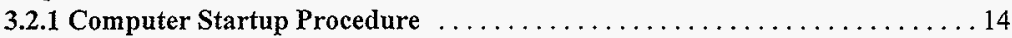

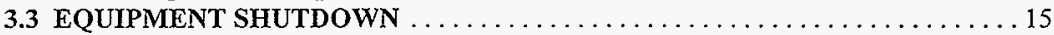

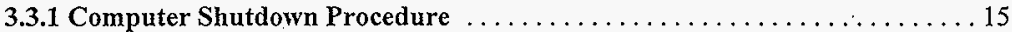

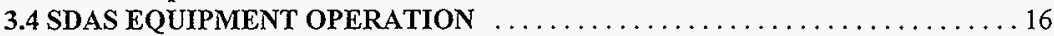

3.4.1 Supervisory Data Acquisition System Top Level Control Panel ........ 16

3.4.1.1 System Health Monitor . . . . . . . . . . . . . . . . . . . . 17

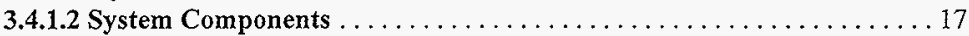

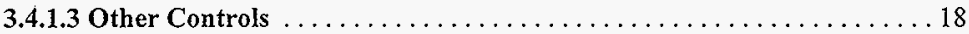

3.4.2 TRIC and Decontamination Control Panel $\ldots \ldots \ldots \ldots \ldots \ldots \ldots \ldots \ldots \ldots$

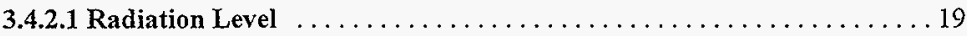

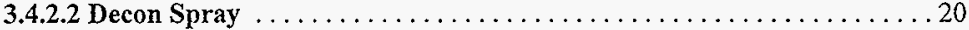

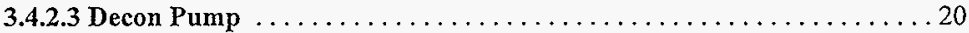

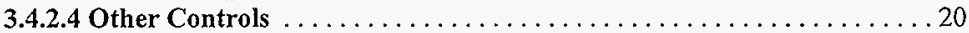

3.4.3 Video Display and Recording Control Panel $\ldots \ldots \ldots \ldots \ldots \ldots \ldots \ldots 21$

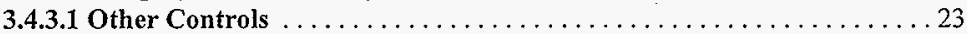

3.4.4 Video Cassette Recorder (VCR) Control Panel ................. 24

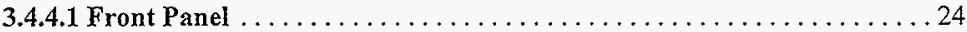

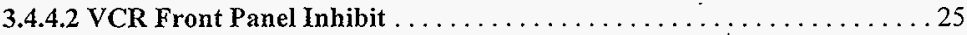

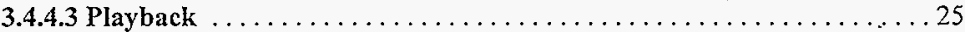

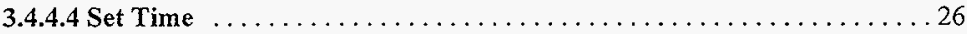

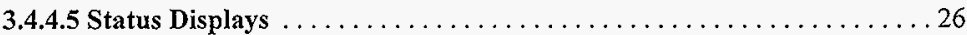

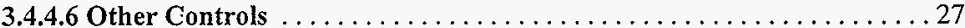

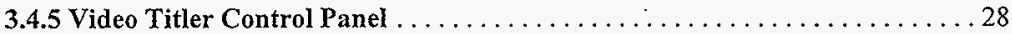

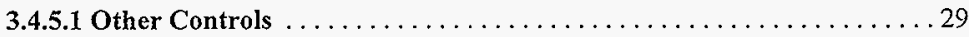

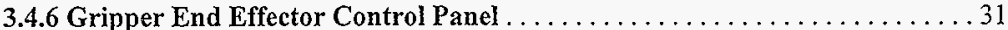

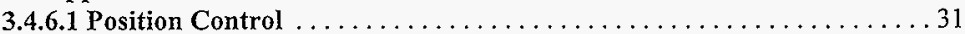

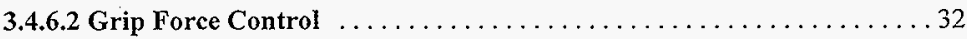

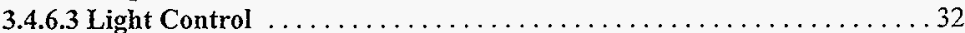

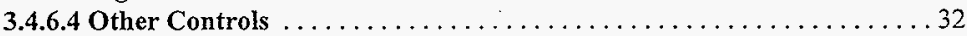

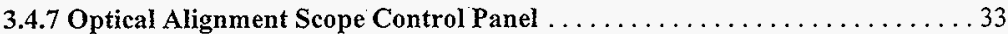

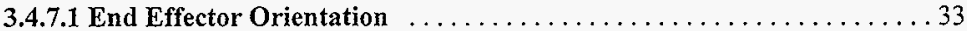

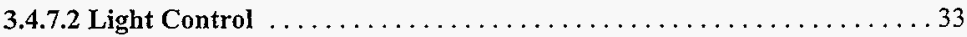

3.4.7.3 Camera Control ............................... 34

3.4.7.4 Laser Control ...................................... 34

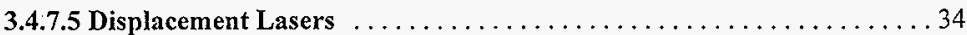

3.4.7.6 Other Controls . ................................ 35

3.4.8 High Resolution Stereo Video System Control Panel .............. 36 


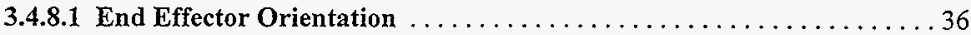

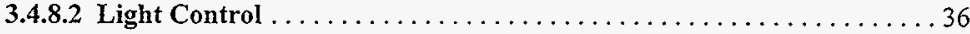

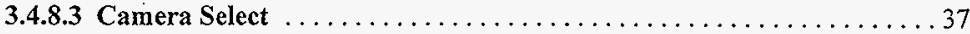

3.4.8.4 Lens Turret Control ............................. 37

3.4.8.5 Closeup Control ................................... 37

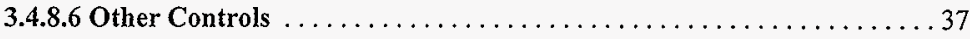

3.4.9 High Resolution Stereo Video System \#2 Control Panel ............. 39

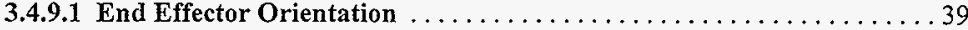

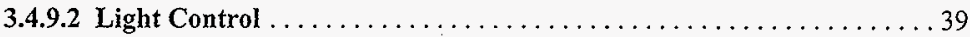

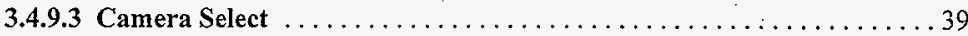

3.4.9.4 Lens Turret Control . . . . . . . . . . . . . . . . . . . . . 40

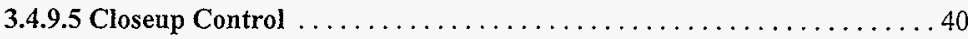

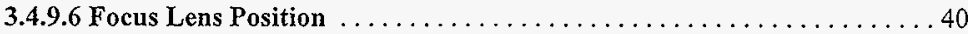

3.4.9.7 Other Controls . ............................ 40

3.4.10 Lens Turret Position Adjustment Control Panel ...............41

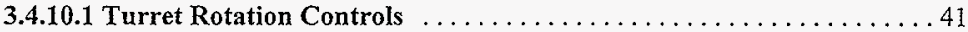

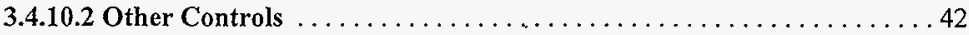

3.4.11 Robot Position Display Panel ........................ 43

3.4.11.1 Cartesian Coordinates ......................... 43

3.4.11.2 Joint Coordinates ............................ 43

3.4.11.3 Tool Interface Plate Identification Values . . . . . . . . . . . 44

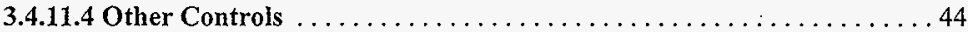

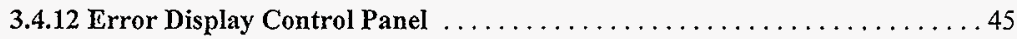

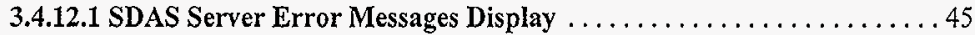

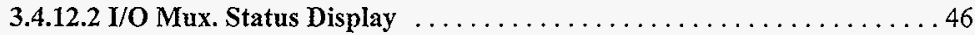

3.4.12.3 Internal Server Status Indicators $\ldots \ldots \ldots \ldots \ldots \ldots \ldots \ldots \ldots$

3.4.12.4 Other Controls .............................. 47

3.4.13 Supervisory Data Acquisition System Top Level Test Control Panel . . . . 48

3.4.13.1 System Health Monitor . . . . . . . . . . . . . . . . . . . 48

3.4.13.2 System Components . . . . . . . . . . . . . . . . . . . . 49

3.4.13.3 Other Controls .............................. 49

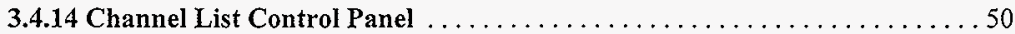

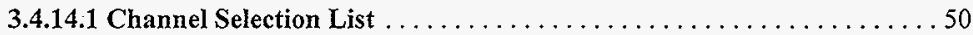

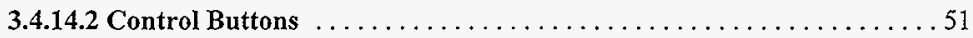

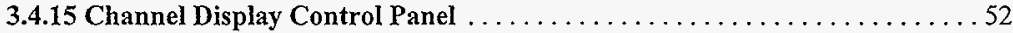

3.4.15.1 Channel Display . . . . . . . . . . . . . . . . . . . . . 53

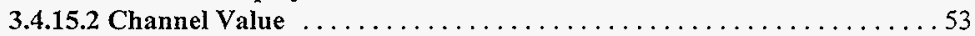

3.4.15.3 Other Controls ............................. 54

3.4.16 Supervisory Data Acquisition System Guest Top Level Control Panel . . . . . 54

3.4.16.1 System Health Monitor . . . . . . . . . . . . . . . . . . . 55

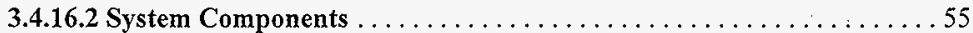

3.4.16.3 Other Controls ................................ 56

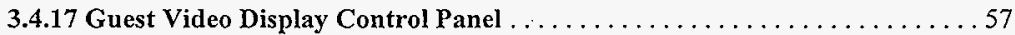

3.4.17.1 Other Control Buttons ............................ 58

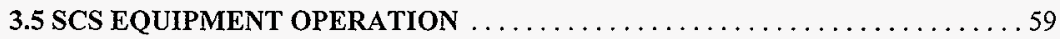

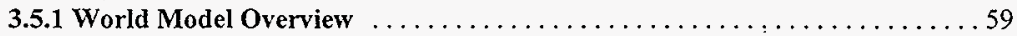

3.5.2 World Model Installation . . . . . . . . . . . . . . . . . . . . . . . . 59 


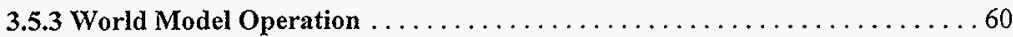

3.5.3.1 Starting the World Model . . . . . . . . . . . . . . . . . . . 60

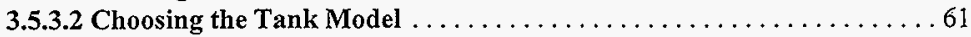

3.5.3.3 Orienting the LDUA to the World Model $\ldots \ldots \ldots \ldots \ldots \ldots \ldots \ldots 6$

3.5.3.4 Adding or Changing the End Effector $\ldots \ldots \ldots \ldots \ldots \ldots \ldots \ldots \ldots$

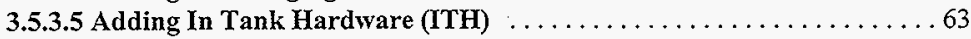

3.5.3.6 Removing or Changing In Tank Hardware $\ldots \ldots \ldots \ldots \ldots \ldots \ldots 6$

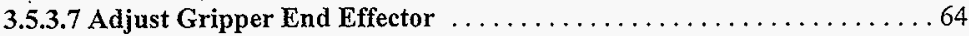

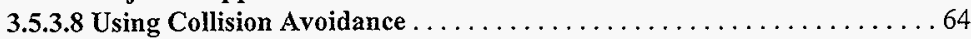

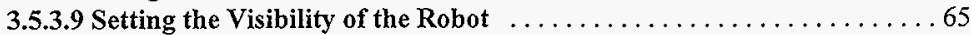

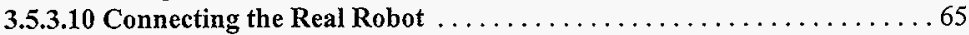

3.5.3.11 Connecting the Simulated Robot ..................... 65

3.5.3.12 Determining Robot Position in World Coordinates ...........66 66

3.5.3.13 Displaying the Force Moment Sensor Data ...............66 66

3.5.3.14 Loading Saved Tank Setups . . . . . . . . . . . . . . . . 66

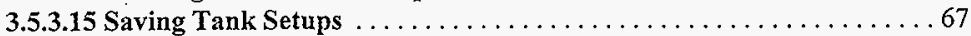

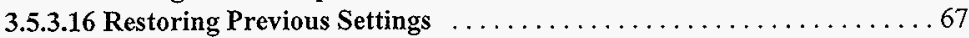

3.5.4 Modifying the World Model $\ldots \ldots \ldots \ldots \ldots \ldots \ldots \ldots \ldots \ldots \ldots \ldots \ldots$

3.5.4.1 Creating New Tank Models $\ldots \ldots \ldots \ldots \ldots \ldots \ldots \ldots \ldots \ldots 6$

3.5.4.2 Creating New In Tank Hardware Models . . . . . . . . . . . . . . . . 69

3.5.4.3 Creating New End Effector Models . . . . . . . . . . . . . . . 69

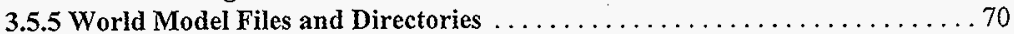

3.5.5.1 World Model Directory Structure $\ldots \ldots \ldots \ldots \ldots \ldots \ldots \ldots \ldots \ldots$

3.5.5.2 World Model Macro Files $\ldots \ldots \ldots \ldots \ldots \ldots \ldots \ldots \ldots \ldots \ldots, \ldots \ldots \ldots$

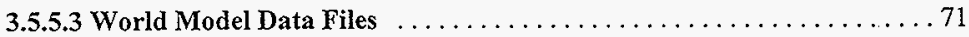

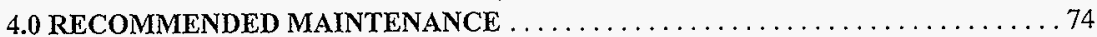




\section{LIST OF FIGURES}

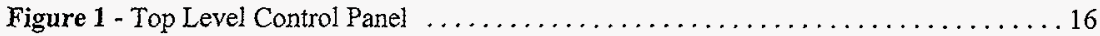

Figure 2 - TRIC and Decontamination Control Panel .......................... 19

Figure 3 - Video Display and Recording Control Panel . . . . . . . . . . . . . . . . . . 21

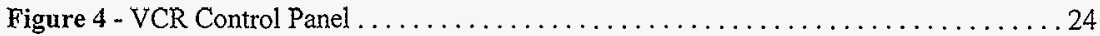

Figure 5 - Video Titler Control Panel ................................. 28

Figure 6 - Gripper End Effector Control Panel ........................... 31

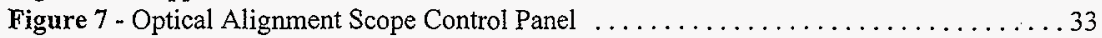

Figure 8 - High Resolution Stereo Video System Control Panel .................... 36

Figure 9 - High Resolution Stereo Video System Control Panel .................... 39

Figure 10 - Lens Turret Position Adjustment Control Panel ................... 41

Figure 11 - Robot Position Display Panel ............................ 43

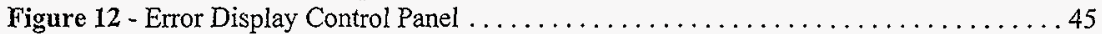

Figure 13 - Top Level Diagnostic Control Panel $\ldots \ldots \ldots \ldots \ldots \ldots \ldots \ldots \ldots \ldots$

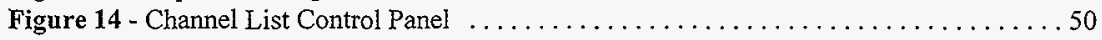

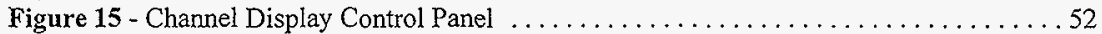

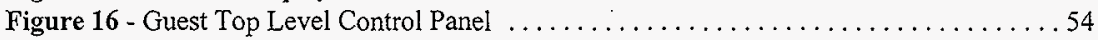

Figure 17 - Guest Video Display Control Panel ....................... 57

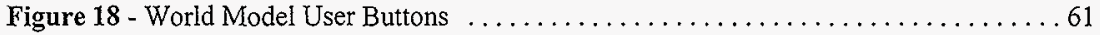

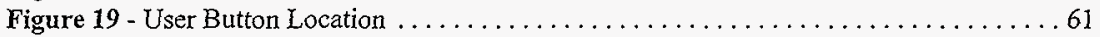

Figure 20 - Choose Tank Pop-up ................................... 61

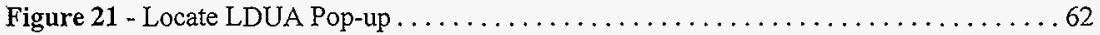

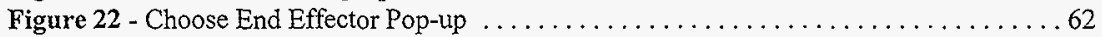

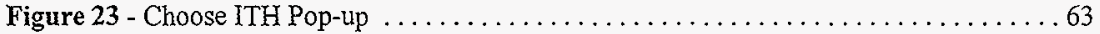

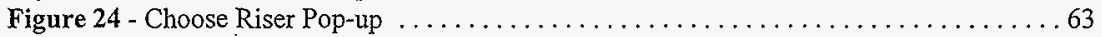

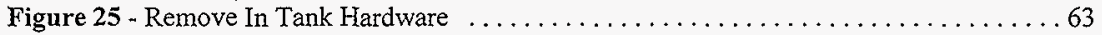

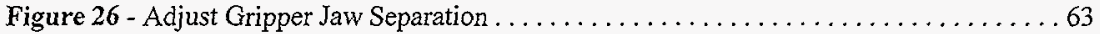

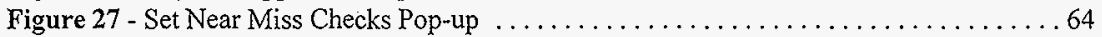

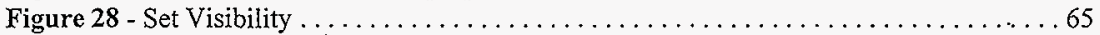

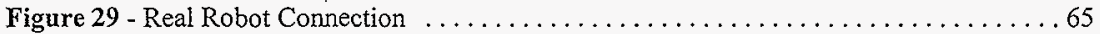

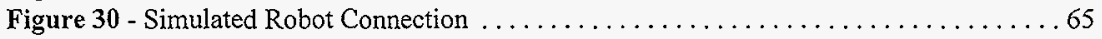

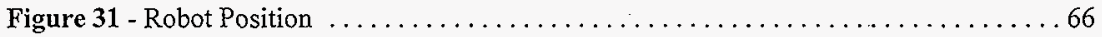

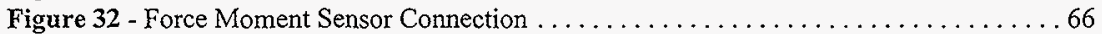

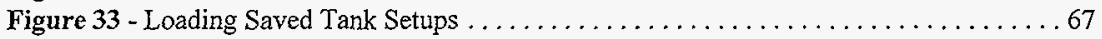

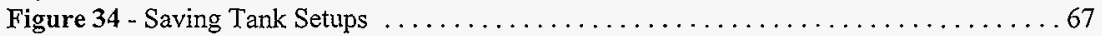

\section{LIST OF FILE LISTINGS}

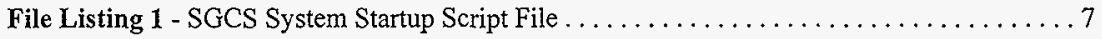

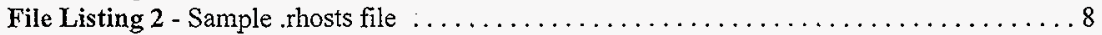

File Listing 3 - Open Windows Initialization File $\ldots \ldots \ldots \ldots \ldots \ldots \ldots \ldots \ldots \ldots$

File Listing 4 - Operator Account Login Script File ....................... 9

File Listing 5 - Operator Account $C$ Shell Initialization File $\ldots \ldots \ldots \ldots \ldots \ldots \ldots \ldots \ldots$

File Listing 6 - Time RPC Server System Startup Script File $\ldots \ldots \ldots \ldots \ldots \ldots \ldots \ldots$ 


\subsection{INTRODUCTION}

This document defines the requirements applicable to the operation, maintenance and storage of the Supervisory Control and Data Acquisition System (SCADAS) and Control Network in support of Light Duty Utility Arm (LDUA) operations at the Hanford nuclear reservation, located near Richland, WA. The SCADAS is subsystem 4200 of the LDUA. The Control Network is subsystem 4400 of the LDUA.

\subsection{DOCUMENT PREREQUISITES}

This document does not provide the reader with a complete understanding of the information required to operate the LDUA system. The equipment operations manuals listed in the reference documents section are required reading. These operations manuals contain important safety and operations limits information that is not included here.

\subsection{SYSTEM OVERVIEW}

The LDUA system is designed to be operated remotely by two operators in the Operations Control Trailer using computer workstations. The LDUA operator is responsible for operating the deployment subsystems (VPM, LDUA, MDS, etc.), and the end effector operator is responsible for operating the end effectors and collecting data. A safety feature (point-ofcontrol) of the robot control system prevents simultaneous control of the robot from the Operations Control Trailer and local panels.

The Supervisory Control and Data Acquisition System is divided into the following subsystems:

System

4200

4210

4220

4230

4400
Acronym

SCADAS

SCS

SDAS

VDRS

CN
Title

Supervisory Control and Data Acquisition System

Supervisory Control System

Supervisory Data Acquisition System

Video Display and Recording System

Control Network

\subsubsection{SUPERVISORY CONTROL SYSTEM}

\subsubsection{The LDUA Console}

The LDUA Console is provided by Spar as part of their system. It operates the LDUA, VPM, and MDS. It provides display and access to the detailed status and operating parameters of the LDUA Subsystem controller. Hand controllers (joysticks) are connected to the LDUA Console for teleoperation of the VPM and LDUA. The primary LDUA Console documentation is 
provided in the Spar LDUA Operations and Maintenance Manual. This document only documents differences in the installation from the Spar manual.

\subsubsection{The SCS Console}

The SCS Console operates in conjunction with the LDUA Console. It provides a 3-D animated graphic display for visualizing and previewing the motion of the VPM and LDUA in the environment of the tank. Motion of the VPM and LDUA can be simulated using any of the motion control modes (teleoperation, autosequences, joint mode, Cartesian mode, etc.) that the LDUA Controller provides. This simulated motion can be used as a preview to check for collisions between the LDUA and the tank structures or waste surface. The LDUA Controller remembers the most recent preview, and the operator can direct it to actually execute the previewed motion.

\subsection{Commercial robotics simulation software}

The robotic simulation software distributed with this system is IGRIP from Deneb Robotics. It is commercial software and is documented in its own manuals.

\subsection{SGCS software}

The Spar SGCS software package is provided by Spar and runs on the SCS Console. The primary SGCS software documentation is provided in the Spar LDUA Operations and Maintenance Manual. This document only documents differences in the installation from the Spar manual.

\subsection{World Model software}

The World Model software package is Hanford specific and is composed of files that customize the behavior of the robotics simulation software. This software is documented in the appropriate sections of this manual.

\subsubsection{SUPERVISORY DATA ACQUISITION SYSTEM}

\subsubsection{The SDAS Console}

The SDAS Console provides the primary user interface for operating end effectors, collecting data, and for monitoring and controlling the TRIC environment subsystems. It provides a single point from which data can be acquired, processed, and stored. The SDAS Console runs LDUA specific software over a commercial data acquisition software package.

\subsubsection{The Guest Console}

The Guest Console provides the ability to obtain information from the system without disturbing the operators at the primary workstations. It is located in the rear area of the Operations Control Trailer away from the operating area. Printers, plotters, and other peripheral devices are also located in this area. 


\subsubsection{The VME Chassis}

The VME Chassis provides a platform for an industry standard embedded processor and data acquisition boards. The embedded processor runs LDUA specific software over a commercial real time operating system.

\subsubsection{Commercial data acquisition software}

The data acquisition software distributed with this system is LabVIEW from National Instruments. It is commercial software and is documented in its own manuals.

\subsubsection{Commercial real time operating software}

The real-time operating software distributed with this system is VxWorks from Wind River Systems. It is commercial software and is documented in its own manuals.

\subsubsection{LDUA specific software}

\subsection{SDAS Server software}

The SDAS Server Software is provided by Sandia National Laboratories. It is documented in the "SDAS Programmer's Manual". This manual documents installation and application details specific to the Hanford LDUA application. A portion of this software called the server runs in the VME embedded processor and provides a data acquisition database that is accessible by many user interface programs acting as clients. Another portion of this software runs on the SDAS and Guest Consoles and provides the client interface for the user interface programs.

\subsection{TopSDAS software}

The TopSDAS software package is Hanford specific and is composed of files that customize the behavior of the commercial data acquisition software. This software is documented in the appropriate sections of this manual.

\subsection{GuestSDAS software}

The GuestSDAS software package is Hanford specific and is composed of files that customize the behavior of the commercial data acquisition software. This software is documented in the appropriate sections of this manual.

\subsubsection{VIDEO DISPLAY AND RECORDING SYSTEM}

There are many video cameras in the LDUA System, including units on the LDUA, in end effectors, and in the ancillary equipment. The Video Display and Recording System provides video monitors and video recorders to display and record pictures from these cameras. All aspects of video switching and display and recording are controlled by the Data Acquisition Workstation, including the video cameras, the routing of signals, the video recorders, and the overlay of titles and other selected information onto the video. 


\subsubsection{CONTROL NETWORK}

The LDUA Supervisory Control and Data Acquisition System is computer based, with several workstations and embedded CPUs that intercommunicate. The Control Network provides a common network for all subsystems to use for this purpose. It provides an 10Base $\Upsilon$ twisted pair Ethernet connection for each computer. The backbone of this network is a twenty-four port hub in the Operations Control Trailer. The Control Network uses standard Hanford Local Area Network (HLAN) components wherever possible so that maintenance and troubleshooting can be accomplished by Hanford network maintenance forces. The Control Network uses standard Category 5 twisted pair wiring which will support the current 10 megabit network and can be upgraded to a 100 megabit network if the extra bandwidth is required. At present, network connections from the ATIE are run from each CPU individually via pairs of fibers in the Fiber Optic Umbilical cable. If necessary to accommodate future growth, another hub could be added in the ATIE to route all its CPU network connections over a single pair of fibers. The hub is intelligent and may be assigned its own network address enabling it to be remotely monitored by Hanford network technicians. While the Operations Control Trailer is located in the Cold Test Facility, the hub is attached to HLAN. When the trailer is placed in the field, it may be operated as a stand alone network or reconnected to HLAN to provide site wide access to LDUA data.

The computers in this document may be referred to by either their functional description or their network names. Below is a table listing the correspondence between functional names, network names, and network addresses.

\begin{tabular}{||l|l|l|l|}
\hline \multicolumn{1}{|c|}{ Computer Functional Name } & $\begin{array}{l}\text { Network } \\
\text { Name }\end{array}$ & $\begin{array}{l}\text { Network } \\
\text { Aliases }\end{array}$ & \multicolumn{1}{|c|}{$\begin{array}{c}\text { Network } \\
\text { Address }\end{array}$} \\
\hline \hline LDUA Console & oboe & Idua3 & 130.97 .64 .14 \\
\hline GISC CPU & cello & vx3Gisc & 130.97 .64 .20 \\
\hline Arm Level Controller CPU & flute & vx3 & 130.97 .64 .21 \\
\hline SDAS Server CPU & bass & & 130.97 .64 .23 \\
\hline SCS Console & fife & & 130.97 .64 .24 \\
\hline SDAS Console & drum & & 130.97 .64 .25 \\
\hline Guest Console & harp & & 130.97 .64 .26 \\
\hline
\end{tabular}




\subsection{REFERENCE DOCUMENTS}

The documents listed below may be consulted as a source of additional information pertaining to the SDAS, its operation, and its maintenance:

1 H-6-14114

2 H-6-14115

3 H-6-14116

4 H-6-14117

5 H-6-14118

$6 \quad H-6-14119$

7 H-6-14120

8 H-6-14121

9 SNL-LDUA-96-006

10 SNL-LDUA-96-009

11 SNL-LDUA-96-010

12 SPAR-LDUA-VDD.065

13 SPAR- LDUA-HBK.056

14 LDUA-OMM-96-6510

15 LDUA-OMM-96-3000

16 WHC-SD-TD-OMM-002

17 WHC-SD-TD-OMM-005

18 LDUA-OMM-96-3300
Control Network Interconnection Diagram

I/O Subsystem Interconnection Diagram

SDAS Cable Diagrams

Connector Adapter Module

Video Display and Recording - Overall Wiring

Diagram

Video Switcher Assembly Details

Video Display and Recording - Cable Assemblies I/O Subsystem - Cable Assemblies

The Supervisory Data Acquisition System (SDAS):

Programmer's Manual

SDAS Programmer's Manual - Appendix A: Device Driver Descriptions

SDAS Programmer's Manual - Appendix B:

Skeleton Device Driver for an Analog Input Record LDUA Software User Document LDUA Operator's Manual

Gripper End Effector Operations and Maintenance Manual

TRIC Operations and Maintenance Manual High Resolution Stereo Video System Operations and Maintenance Manual Common Video End Effector Operations and Maintenance Manual

DECON Operations and Maintenance Manual 
HNF-SD-TD-OMM-001, Rev. 1

Page 6

\subsection{INSTALLATION PROCEDURES}

This section provides instructions for all installation details that are unique to the Hanford LDUA application. The basic installation philosophy is that there will be a single account for the operators to use (oper), and a single account for software installation (ellis). The installed files will be read-only to the operators, and therefore, can not be modified or deleted. Also, the software to be installed is loaded into a central release directory with links to the current version so that you can install a new version and easily switch back to the old version if there are problems with the new version.

A central assumption of this section is that all software components are distributed as compressed tar files. These distribution files reside in a central master repository. There is a tape containing a copy of all these distribution files that may be used to create the repository. All installation procedures assume that the distribution file is copied wherever necessary from the master repository.

\subsection{INSTALLATION OF SCS SOFTWARE}

\subsubsection{INSTALLATION OF LDUA CONSOLE SOFTWARE}

Create release directory as /users/release.

cd /users

mkdir release

Change the owner to ellis, group to staff (15), and the permissions to drwxr-xr-x (755).

chown ellis release

chgrp staff release

chmod 755 release

Copy the new release from the master repository an install it with the commands:

co release

zcat ldua 1.1.0.96.08.16.tar.z I tar xvf -

rm Idua_1.1.0.96.08.16.tar.Z

Create links from new release directory to directory /users/release/current with command: In $-s$ Idua_1.1.0.96.08.16 current

Create a link from the failure messages directory into the oper home directory with command In -s current/fm /users/oper/fm

The rest of the installation procedure is as per the LDUA Software User Document (reference 12). The only exceptions are that the software has been installed in the locations listed above. 


\subsubsection{INSTALLATION OF SCS CONSOLE SOFTWARE}

The installation instructions contained in the LDUA Software User Document (reference 12) are completely replaced by the instructions given here. Copy the SGCS executable file from the LDUA Console current release directory to the SCS Console directory /usr/local with the commands:

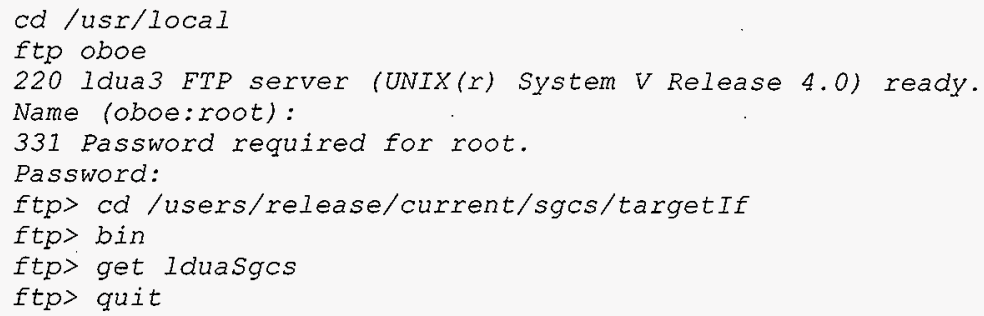

Create a file /etc/init.d/lduaSgcs with the following contents:

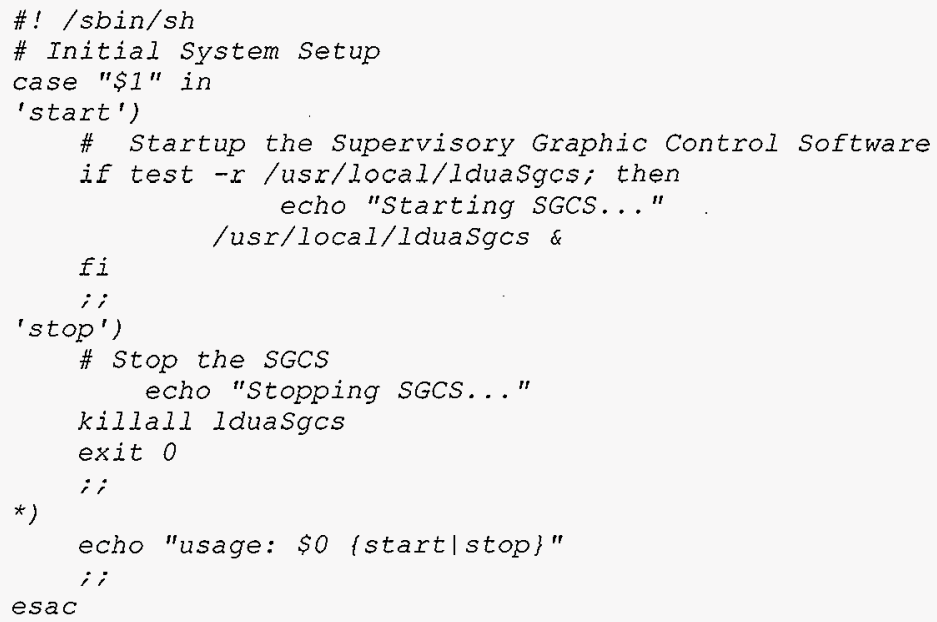

File Listing 1 - SGCS System Startup Script File

Create links in the startup directories with the following commands:

cd letc

In -s rco.d/K30lduaSgos ro2.d/S40lduaSgos init.d/lduasgos

Install the World Model software per the World Model installation section 3.5.2. 


\subsection{INSTALLATION OF SDAS SOFTWARE}

These procedures detail the installation of the software necessary to run SDAS.

\subsubsection{INSTALLATION OF SDAS CONSOLE SOFTWARE}

This procedure explains how to create al the user accounts and install all the software resident in the SDAS Console.

\subsubsection{CREATE VXWORKS ACCOUNT}

Create an account vxworks on the SDAS Console, with account number 1002 and group staff (15). The home directory should be /export/home/vxworks. Create a file /export/home/vxworks/.rhosts which contains a list of all the vxworks hosts which will have to access files belonging to the vxworks account. Here is an example :

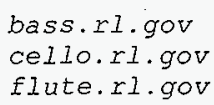

File Listing 2 - Sample .rhosts file

\subsubsection{CREATE OPER ACCOUNT}

Create an account oper on the SDAS Console, with account number 952 and group staff (15). The home directory should be /export/home/oper. Create a file /export/home/oper/openwin-init with the following contents: 


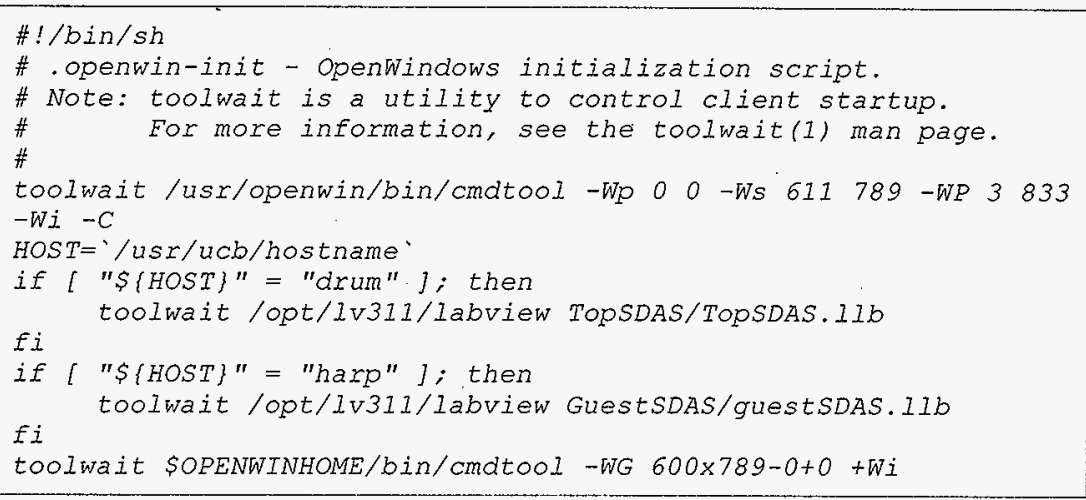

File Listing 3 - Open Windows Initialization File

Make sure that this initialization file is executable with the following command: chmod 755 .openwin-init

Create a file /exporthome/oper/login with the following contents:

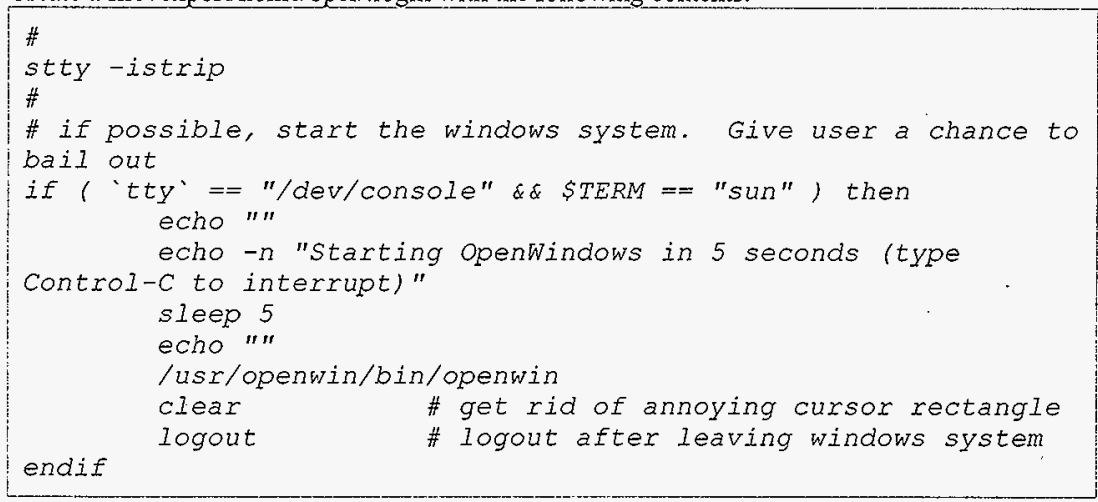

File Listing 4 - Operator Account Login Script File

Create a file/export/home/oper/.cshrc with the following contents: 


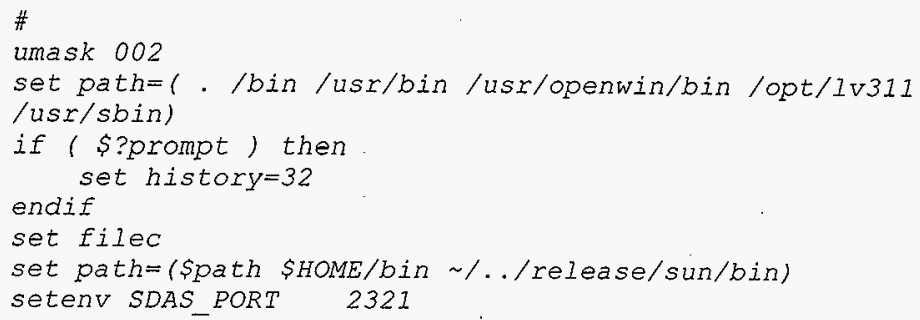

File Listing 5 - Operator Account C Shell Initialization File

\subsubsection{INSTALL SDAS SOFTWARE}

Login to the oper account. Copy the new release from the master repository an install it with the commands:

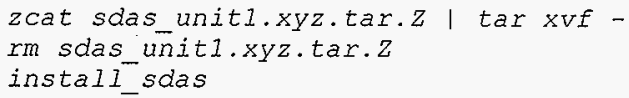

\subsubsection{INSTALL TIME SERVER SOFTWARE}

Create a file /etc/init.d/time_server on the SDAS Console with the contents shown in File Listing

6. Create links to this file in the startup directories with the following command:

In -s /etc/rc2.d/s93time_server /etc/init.d/time_server

Reboot the system.

\subsubsection{INSTALLATION OF GUEST CONSOLE SOFTWARE}

Create an account oper on the Guest Console, with account number 952 and group staff (15). The home directory should be remotely mounted from the SDAS Console directory lexport/home/oper. No further setup of this account is required, because the necessary files were setup as part of the SDAS Console oper account creation. 


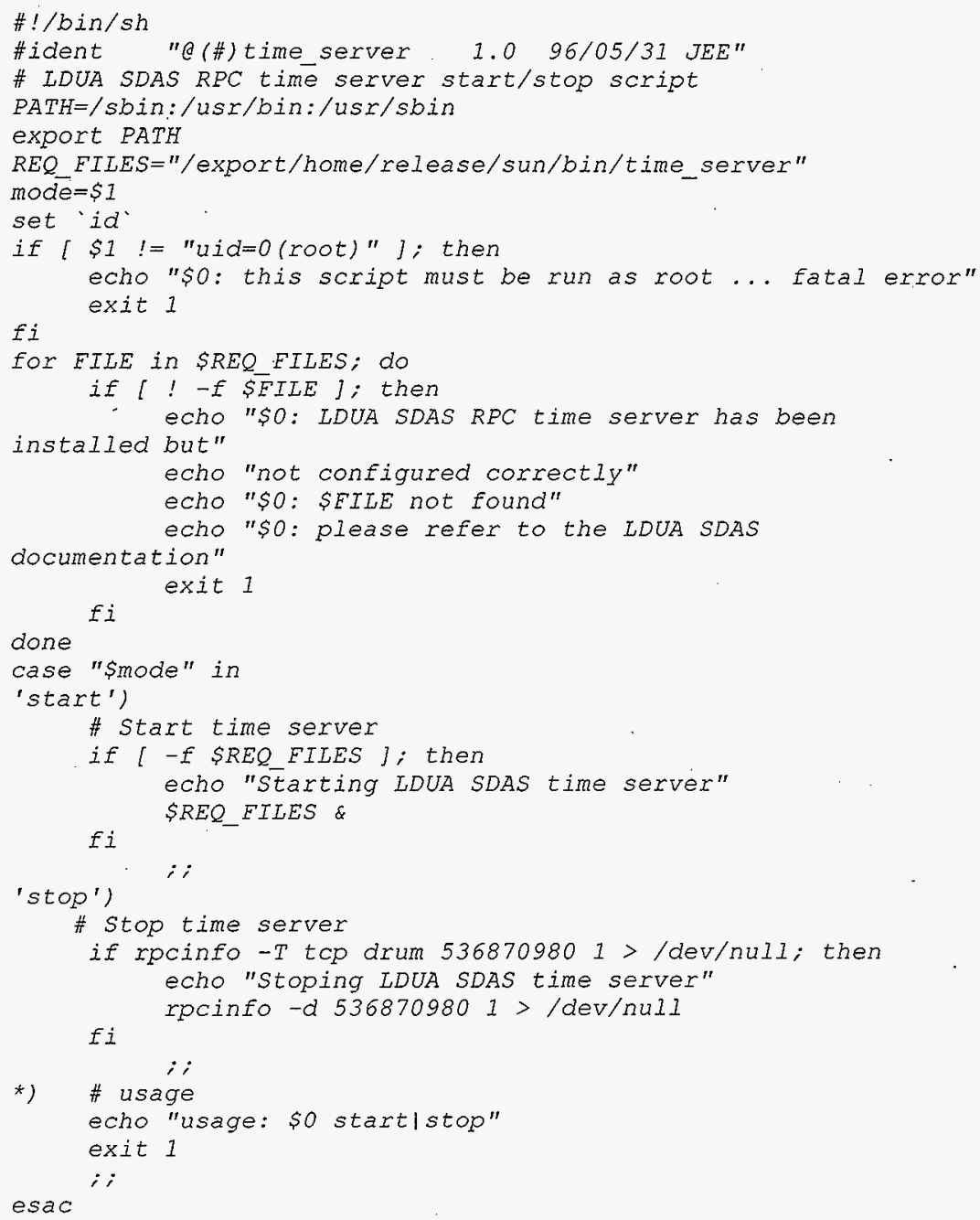

File Listing 6 - Time RPC Server System Startup Script File 


\subsection{CONFIGURATION OF SDAS VME HARDWARE}

These procedures detail the configuration of the VME hardware necessary to run SDAS.

\subsubsection{CONFIGURE SDAS SERVER CPU}

The boot parameters for the SDAS server CPU should be set as follows:

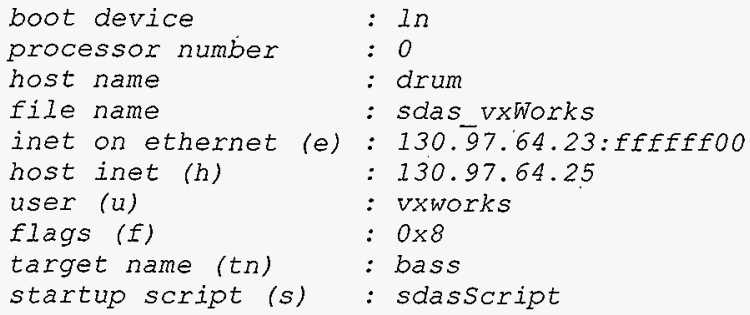

\subsubsection{CONFIGURE GISC CPU}

The boot parameters for the GISC CPU should be set as follows:

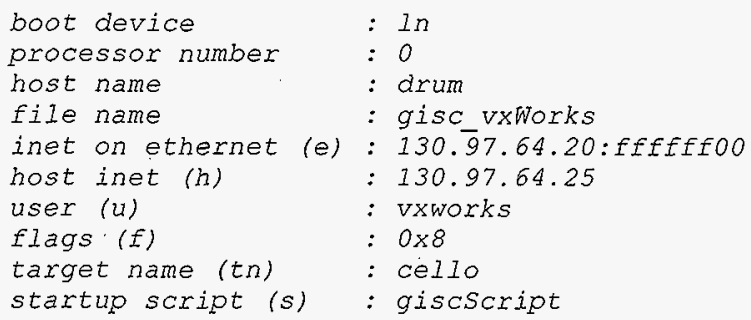

\subsubsection{CONFIGURE ISIO-2 BOARDS}

SDAS supports up to two ISIO-2 eight port serial interface boards. The first board (ports 1-8) is configured at the default address $\$ 960000$. The second board should be configured at the next available address $\$ 980000$. See the ISIO-2 Hardware User's Manual page 5-4 for the details of jumper selection. The software could support more than two boards, but that is what is hard coded. To increase this limit, change the MAXBOARDS value in the file isio.h and recompile the server. Additional configuration information is shown on the I/O Subsystem Interconnection Diagram, (reference 2). 


\subsubsection{CONFIGURE IP CARRIER BOARDS}

SDAS supports up to 32 IP boards in eight IP Carriers. The first IP Carrier board is configured at the default address $\$ 6000$. Each additional carrier's address is higher by $\$ 800$ (i.e., $\$ 6800$, $\$ 7000$, etc.). The CPU-30ZBE accesses the module by adding $\$$ fcffo000, which is why the SDAS Programmer's Manual specifies an address of $\$ f \mathrm{fff} 6000$. The initialization software begins with the first address and searches each successive carrier address until an invalid address is reached. The address checked is the address of the IP module in the A slot. Therefore any carrier which does not have a module in the A slot will terminate the search. See the VIPC610 User Manual, I/O Addressing, page 7 for the details of jumper selection. Additional configuration information is shown on the I/O Subsystem Interconnection Diagram, (reference 2). 


\subsection{OPERATING PROCEDURES}

This section provides instructions for the normal handling and operation of the SDAS in support of routine LDUA activities.

\subsection{EQUIPMENT REMOVAL, STORAGE, AND SETUP}

This section covers the control system equipment removal, storage, and setup required to move Operations Control Trailer. When moving the Operations Control Trailer all equipment that is not permanently mounted in a control rack shall be strapped down or tied down in some manner. The video monitors, computers and peripherals are normally strapped to the desk or shelf on which they are located. The large computer and stereo monitors are disconnected and placed on the floor of the trailer where they can not move should the trailer come to an abrupt stop. They shall be covered or wrapped with moving blankets to prevent damage due to rubbing or bumping each other or the adjacent furniture.

When restoring the system after moving the Operations Control Trailer, simply remove the tie downs and replace and reconnect the monitors before performing the computer startup procedure.

\subsection{EQUIPMENT STARTUP}

\subsubsection{Computer Startup Procedure}

This procedure applies to all the UNIX computer systems. It may be used to startup any or all of the systems, by applying the appropriate sections. When starting up these systems, please take into account the following file server relationships:

\begin{tabular}{|l|l|}
\hline \multicolumn{1}{|c|}{ File server } & \multicolumn{1}{c|}{ Clients } \\
\hline \hline drum & bass, cello, fife, harp \\
\hline oboe & fife, flute \\
\hline
\end{tabular}

To startup any of the Sun computer systems, turn on the power. Wait for the system to startup. The system is ready when the logon prompt is displayed. The logon prompt looks like:

host-name console login:

To startup the SGI computer system, turn on the power. Open the front panel and press the power switch. The SGI computer will automatically perform a proper startup sequence.

To startup any of the VME computer systems, turn on the power. Wait for the system to startup. If the VME computer starts up before it's file server, reset the VME crate. The computers in the 
VME crate load their software from their file server. Resetting the crate tells the VME computers to reload their software.

\subsection{EQUIPMENT SHUTDOWN}

\subsubsection{Computer Shutdown Procedure}

This procedure applies to all the UNIX computer systems. It may be used to shutdown any or all of the systems, by applying the appropriate sections. When shutting down these systems, please take into account the following file server relationships:

\begin{tabular}{|l|l|}
\hline \multicolumn{1}{|c|}{ File server } & \multicolumn{1}{|c|}{ Clients } \\
\hline \hline drum & bass, cello, fife, harp \\
\hline oboe & fife, flute \\
\hline
\end{tabular}

Note: Shutting down these systems by simply turning off the power risks corrupting the data stored on the hard disk. If the system is quiet and there is no disk activity, the risk is small. If the system is active and there is a great deal of disk activity, the risk of corruption is very great. Please power down without shutting down only as a last resort under dire consequences!!!

Shutdown all application software and logoff all user accounts for each system to be shutdown.

To shutdown the SGI computer, open the front panel and press the power switch. The SGI computer will automatically perform a proper shutdown sequence.

To shutdown any of the Sun computers, perform the following steps for each computer.

Enter a logon name:

login: halt

Enter the password:

Password:

Wait for the system to shutdown. This may take several minutes. The system is shutdown when the following messages are displayed:

The system is down.

syncing file systems... [8] [5] done

Program terminated

Type help for more information

OK.

Turn off the power to the computer systems by shutting off the power strip. 


\subsection{SDAS EQUIPMENT OPERATION}

\subsubsection{Supervisory Data Acquisition System Top Level Control Panel}

This panel is the main SDAS control panel as shown in Figure 1. This panel controls and displays server connection, monitors system health, controls access to system components and the data $\log$.

Server connection is made automatically when TopSDAS is run. If a connection can not be made a message will be displayed and the control panel will be terminated. Once a connection is made the server is disconnected by selecting the Quit $S D A S$ button which requires user verification before disconnecting. The Quit SDAS button also terminates execution.

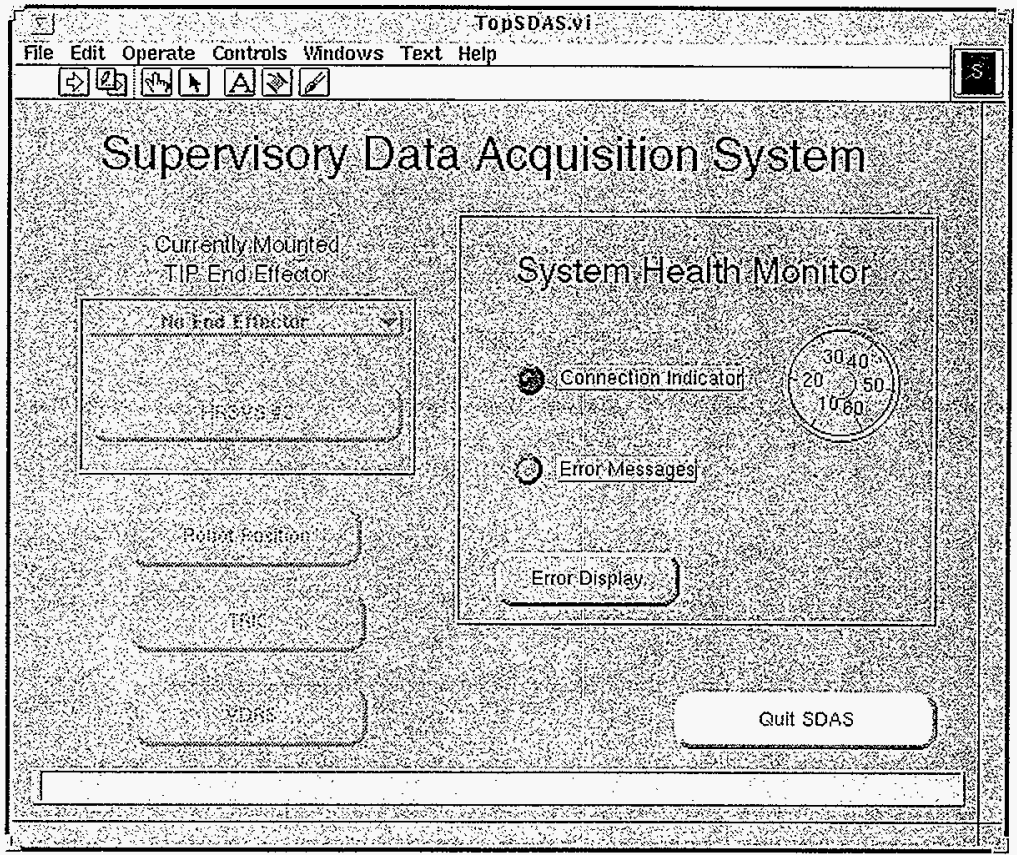

Figure 1 - Top Level Control Panel 


\subsubsection{System Health Monitor}

The System Health Monitor section of the panel display includes the server connection, activity gauge, error message indicator, and error display button.

Connection Indicator - The indicator tells the user if a connection to the server has been made. This indicator is green when a connection exists and red when a connection does not exist. Lack of communication is a fatal condition.

Activity Gange - A gauge shows whether there is data flowing between the server and the control panel. This gauge will rotate when a connection exists and will stop when the control panel is no longer running or if there is condition inhibiting data flow between the server and the Control panel. Lack of data flow may indicate either a fatal condition or a performance problem.

Error Message Indicator - The indicator tells the user that server error message have been posted and may be observed on the Error Display Control Panel. If there are any unacknowledged error messages, this light is yellow.

Error Display Button - The button activates the Error Display Control Panel. Unacknowledged messages are acknowledged and the current error display is cleared.

Current Error Display - The display window at the bottom of the control panel shows the most recent unacknowledged message.

\subsubsection{System Components}

System components are accessed via control buttons. Controls that require a connection are greyed-out until a server connection is made. Each system component has its own display panel that is displayed by selecting the corresponding control button. System components that have not been integrated with SDAS will display an error message instead of the specified control panel. The controls are:

Robot Position - Activates the Robot Position display panel. This control is grayed out if robot position information is not available.

Currently Mounted TIP End Effector - The currently mounted TIP end effector may be either automatically determined from information gotten from the robot or selected from a pull-down menu. This control is grayed out and the label changes automatically with changes of the end effector, if robot position information is available.

Once the currently mounted TIP end effector has been selected a control button for that end effector is enabled. This control button activates a control panel for the selected end effector.

The mounted TIP end effector may supply a video source to the Video Display and Recording System. The settings on both the TopSDAS control panel and the VDRS control panel must be compatible for correct operation. Changing one might require changing the other as not all end 
effector types are compatible with all video destinations. The user will be alerted when a setting has changed unless both the previous and new end effector do not supply a video source.

TRIC - Activates the TRIC and Decontamination System control panel.

VDRS - Activates the Video Display and Recording System control panel.

\subsubsection{Other Controls}

Quit SDAS - Closes any open panels except this panel, disconnects the server and terminates execution. This button requires user verification. 


\subsubsection{TRIC and Decontamination Control Panel}

This panel is the control panel for the Tank Riser Interface and Confinement radiation detectors and the Water Decontamination system as shown in Figure 2. It includes radiation levels, decon spray, decon pump, and riser isolation valve controls as well as other controls.

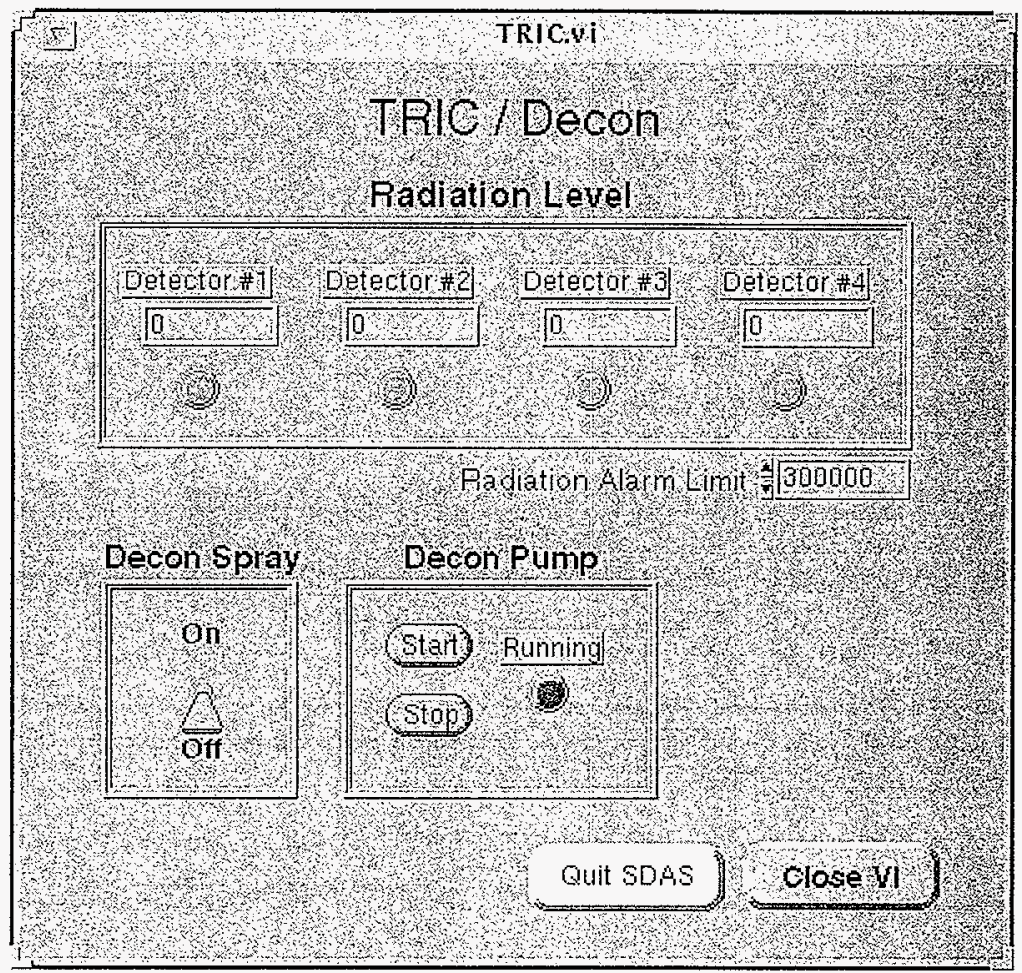

Figure 2 - TRIC and Decontamination Control Panel

\subsubsection{Radiation Level}

Detector - Detectors 1 through 4 display number counts $(0-350,000)$, see instrument calibration data to convert counts to radiation levels.

Alarm Light - Alarm lights 1 through 4 are green if detector is lower than the limit, red if higher. When any one of the alarm lights turn red, an inhibit signal is generated that prevents the mast from moving up.

Limit - Limit is a user-setable alarm limit $(0-350,000)$ counts. 


\subsubsection{Decon Spray}

On/Off - Water sprays as long as switch is on and the pump is running.

\subsubsection{Decon Pump}

Start - Starts the decon pump running. This is a momentary switch that closes the motor contactor

Stop - Stops the decon pump running. This is a momentary switch that closes the motor contactor.

Running - Shows whether the pump is running. The light is off when the motor contactor is open and on when it is closed.

\subsubsection{Other Controls}

Close VI. - Closes this Control Panel and returns control to the calling VI. If any-sub-vi's for this panel are still open, they must be closed before this panel can close.

Quit SDAS - Closes this and all other open panels except TopSDAS, disconnects the server and terminates VI execution. This button requires user verification. 


\subsubsection{Video Display and Recording Control Panel}

This VI is the main Video Display and Recording control pane as shown in Figure 3. The user can select video sources for the available video destinations, except video monitor 5 and stereo monitor 2, access the VCR \& Titler control panels, and print the display on Monitor 3. There is a companion VI that controls Video monitor 5 and Stereo Monitor 2 and only displays the rest of the settings. See Guest VD Control Panel.

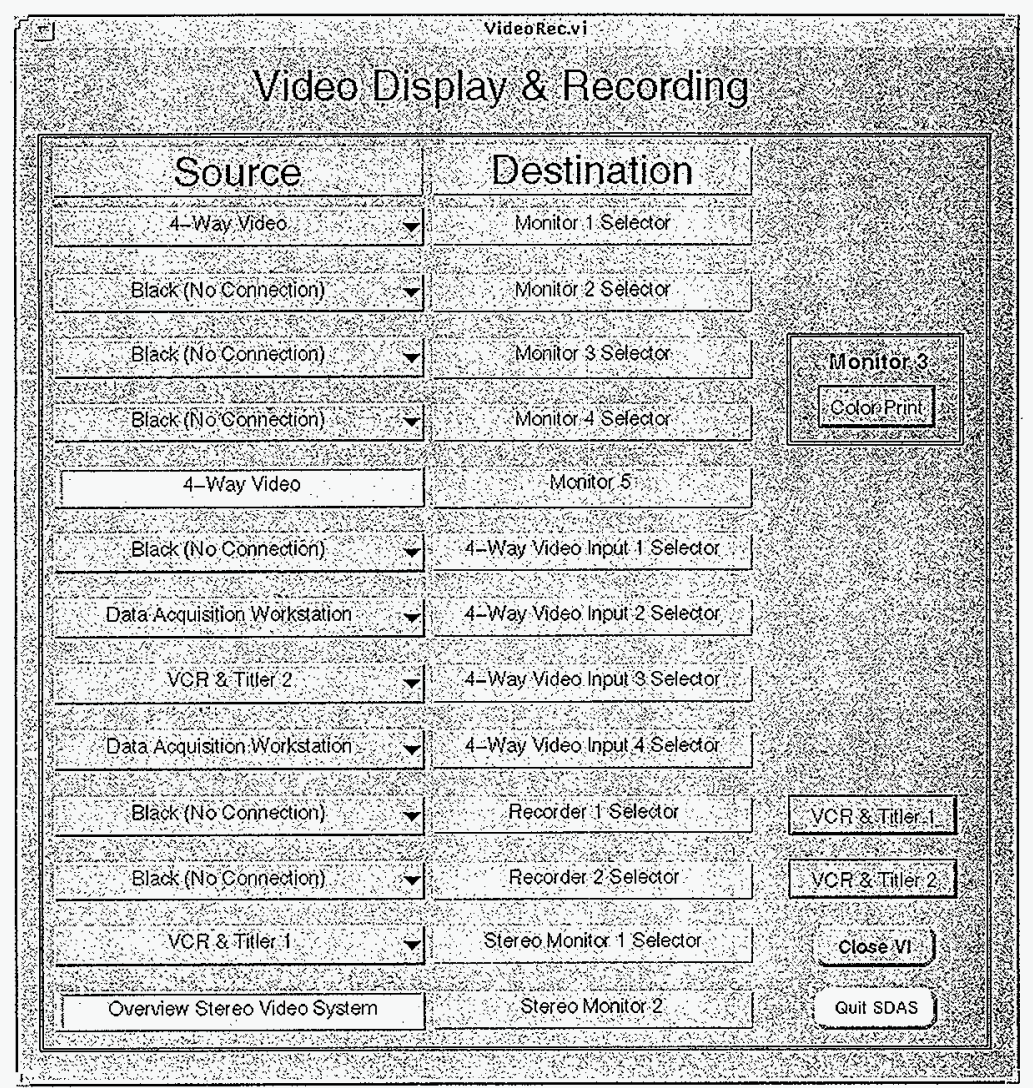

Figure 3 - Video Display and Recording Control Panel

Each destination consists of a label and either a pull-down menu or a simple display of possible source settings for that destination. The menus are unique to each destination or destination type, allowing connections to only those sources that are sensible. 
The Mounted TIP End Effector, Left TIP Camera, and Right TIP Camera sources refer to the current end effector mounted on the system. The mounted TIP End Effector is set from TopSDAS. These settings must be compatible for correct operation. Changing one might require changing the other as not all end effector types are compatible with all destinations. The user will be warned when a change does not meet the minimum requirements for operation. The minimum requirements are defined below for each source/destination combination.

There are five video monitors. The display source for the first four monitors is controlled from this panel using pull-down menus. The pull-down menus for these monitors are identical. The display source for the fifth monitor will be shown on this panel but can not be controlled. The possible sources are:

\section{4-Way Video}

Black (no connection)

Data Acquisition Workstation

Left Tip Camera - Minimum requirements are that the end effector has a left camera.

Mounted TIP End Effector - Minimum requirements are that the end effector has s-video. Operations Workstation

Right Tip Camera - Minimum requirements are that the end effector has a right camera.

Shoulder Camera

Side by Side Stereo

Overview Video

Operations Overview Video

VCR \& Titler 1

VCR \& Titler 2

There is a 4-Way Video that shows four different views or inputs on a single display. The pulldown menus for each view are identical. The possible sources are:

Black (no connection)

Data Acquisition Workstation

Left Tip Camera - Minimum requirements are that the end effector has a left camera.

Mounted TIP End Effector - Minimum requirements are that the end effector has s-video.

Operations Workstation

Right Tip Camera - Minimum requirements are that the end effector has a right camera.

Shoulder Camera

Side by Side Stereo

Overview Video

Operations Overview Video

$V C R \&$ Titler 1

VCR \& Titler 2

There are two video recorders each with its own titler. The pull-down menus for each recorder are identical except for the VCR \& Titler selections. Each recorder allows the other as a source, it does not allow itself. The possible sources are: 
Black (no connection)

Data Acquisition Workstation

Left Tip Camera - Minimum requirements are that the end effector has a left camera.

Mounted TIP End Effector - Minimum requirements are that the end effector has s-video.

Operations Workstation

Right Tip Camera - Minimum requirements are that the end effector has a right camera.

Shoulder Camera

Side by Side Stereo

Overview Video

Operations Overview Video

VCR \& Titler 1 (Recorder 2 only)

VCR \& Titler 2 (Recorder 1 only)

There are two Stereo Monitors. The display source for the first monitor is controlled from this panel using pull-down menus. The display source for the second monitor will be shown on this panel but can not be controlled. Some sources require that the signal first pass through a stereo converter before being sent to the Stereo Monitor. This conversion is invisible to the user. The possible sources are:

Black (no connection)

Mounted TIP End Effector - Minimum requirements are that the end effector has stereo Overview Stereo Video

VCR \& Titler 1

VCR \& Titler 2

\subsubsection{Other Control Buttons}

Color Print - Prints the display on monitor \#3.

VCR \& Titler 1 - Activates the video control panel for the Sony VCR \#1, which also provides access to the titler $\# 1$.

VCR \& Titler 2 - Activates the video control panel for the Sony VCR \#2, which also provides access to the titler \#2.

Close VI - Closes this control panel and returns control to the calling VI. If any sub-vi's for this panel are still open they must be closed before this panel can close.

Quit SDAS - Closes this and all other open panels except TopSDAS, disconnects the server and terminates VI execution. This button requires user verification. 


\subsubsection{Video Cassette Recorder (VCR) Control Panel}

This panel is the control panel for a SONY Model SVO-9600 VCR as shown in Figure 4. It consists of Front Panel, Playback, Set Time and Status sections, and provides access to the corresponding titler control panel.

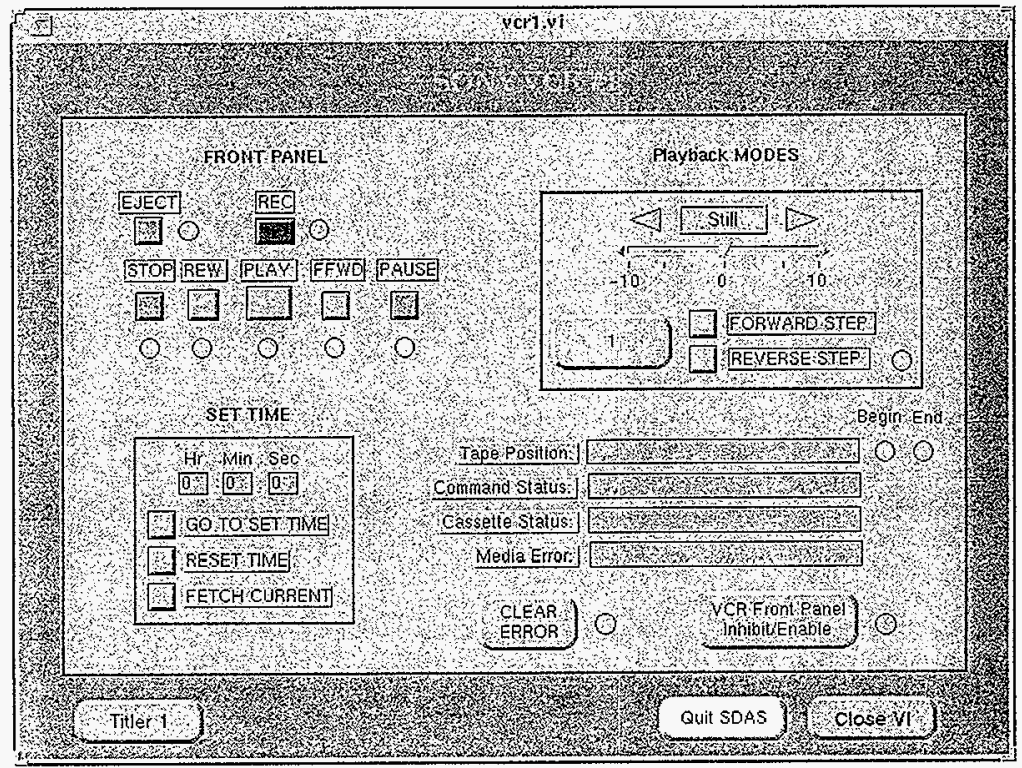

Figure 4 - VCR Control Panel

\subsubsection{Front Panel}

The Front Panel section consists of seven buttons corresponding to the controls found on the actual VCR front panel. All buttons have a corresponding indicator that shows when the control is activated.

EJECT - Ejects the tape from the VCR. The EJECT indicator is green while ejecting.

FFWD - Fast forwards the tape. The FFWD indicator is green while the tape is fast forwarding.

PAUSE - There are two pause modes: REC PAUSE and PLAY PAUSE. When the VCR is in either PAUSE mode for an extended time, the VCR will revert to a long pause state and the PAUSE indicator will be yellow. 
REC PAUSE occurs when PAUSE is selected while the VCR is recording. When in REC PAUSE mode the REC indicator is yellow and both the PLAY and PAUSE indicators are green.

PLAY PAUSE occurs when PAUSE is selected while the VCR is performing any function except REC. When in PLAY PAUSE mode both the PLAY and PAUSE indicators are green.

Selecting PAUSE when the VCR is in REC PAUSE mode causes the VCR to continue recording.

Selecting PAUSE when the VCR is in PLAY PAUSE mode cases the VCR to resume the function that was active when $P A U S E$ was selected.

PLAY - Plays the tape unless the VCR is in REC PAUSE mode. If the VCR is in REC PAUSE mode recording resumes. The PLAY indicator is green while the tape is playing, recording, or paused.

$R E C$ - Displays a message box asking the user to confirm that recording is desired unless the VCR is currently in REC PAUSE mode. After user confirmation, recording begins. The REC indicator is yellow while recording and the PLAY indicator is green.

$R E W$ - Rewinds the tape. The REW indicator is green while the tape is rewinding.

STOP - Stops the current tape transport and turns the corresponding indicator green.

\subsubsection{VCR Front Panel Inhibit}

There are two methods for controlling the VCR. The VCR can be controlled using the SDAS remote control panel or the VCR local front panel. Only one method can be enabled at a time. The default method is via the SDAS remote control panel

VCR Front Panel Inhibit/Enable - This switch allows the user to enable or inhibit the VCR local front panel.

When the VCR local Front Panel is inhibited, all VCR controls except EJECT are disabled, all SDAS control panel controls are enabled, and the indicator is green.

When the VCR local Front Panel is enabled, all VCR controls are enabled, all SDAS control panel controls are disabled/grayed-out, and the indicator is yellow.

\subsubsection{Playback}

The Playback section supports Forward and Reverse Shuttle at all speeds and Forward and Reverse Step. It consists of a tape speed and direction selector, an indicator light, and control buttons.

Speed and Direction - This is a slide selector that lets the user set the tape speed and direction for playback. The available directions are reverse and forward. 
The speeds available are:

$\begin{array}{ll}0 & \text { still } \\ 1 / 30 & 1 / 30 \times \text { normal speed } \\ 1 / 10 & 1 / 10 \times \text { normal speed } \\ 1 / 5 & 1 / 5 \times \text { normal speed } \\ 1 / 2 & 1 / 2 \times \text { normal speed } \\ 1 & 1 \times \text { normal speed } \\ 2 & 2 \times \text { normal speed } \\ 6 & 6 \times \text { normal speed } \\ 10 & 10 \times \text { normal speed }\end{array}$

$<$ current playback direction and speed > [ NAME THIS BUTTON AND DESCRIBE THE VARIOUS LABELS IT MAY HAVE]

This control button moves the tape in the direction and at the speed selected using the Speed and Direction Selector. The indicator is green when there is any playback activity [DESCRIBE INDICATOR \&/OR NAME IT \&/OR MOVE IT UNDER THE BUTTON.]

FORWARD STEP - When in PAUSE mode it advances the tape one frame then returns the VCR to PAUSE mode. The frame number in the tape position display will advance one frame. In all other modes it puts the VCR in PLAY PAUSE mode.

REVERSE STEP - When in PAUSE mode it reverses the tape one frame then returns the VCR to PAUSE mode. The frame number in the tape position display will reverse one frame. In all other modes it puts the VCR in PLAY PAUSE mode.

\subsubsection{Set Time}

The Set Time section consists of four controls.

Set Time - An input control field that allows you to select a tape transport time in $<$ hours $>:<$ minutes $>:<$ seconds $>$.

GO TO SET TIME - Advance or rewind the tape until the tape position display time matches the time specified in the Set Time control and switch to PLAY PAUSE mode when done. The FFWD or REV indicator is green while the tape is moving. The Tape Position Status displays the tape movement.

RESET TIME - Reset the time in the tape position display to 0:00:00. This does not modify the Set Time control.

FETCH CURRENT - Sets the current tape position into the Set Time Control.

\subsubsection{Status Displays}

The Status section consists of four display windows, two indicators and a control button. 
The display windows are:

Tape Position - Displays the current tape transport time in $\langle$ hours $\rangle:<$ minutes $\rangle:\langle$ seconds $\rangle$ $<$ frame number $>$.

Command Status - Displays the current command status string returned from the VCR.

Cassette Status - Displays the status of the cassette. One of the following strings will be dispiayed based on the returned status: Tape Out, Tape In, or Tape In and Write Protected.

Media Error - Displays any media errors. One of the following strings will be displayed based on the returned status: No Error, Slack Detect, Dew Detect, or Unknown Error [REFER TO USERS MANUAL FOR BETTER EXPLANATION OR COME UP WITH ONE!].

The indicators are:

Tape Begin - This indicator is green when current tape position is at the beginning of the tape. This only occurs for a brief moment when the tape hits the end of travel before shutting off.

Tape End - This indicator is green when current tape position is at the end of the tape. This only occurs for a brief moment when the tape his the end of travel before shutting off.

Error Indicator - This indicator is red when there is an error, otherwise it is green. and control button:

CLEAR ERROR - Clears the last transferred numerical command and error conditions.

\subsubsection{Other Control Buttons}

Titler $\langle n\rangle-$ Activates the titler control panel for this VCR.

Close VI - Closes this control panel and returns control to the calling VI. If any sub-vi's for this panel are still open they must be closed before this panel can close.

Quit SDAS - Closes this and all other open panels except TopSDAS, disconnects the server and terminates VI execution. This button requires user verification. 


\subsubsection{Video Titler Control Panel}

This is the control panel for the video titlers as shown in Figure 5. It includes a Simulated Display, Text Display options, and other controls. Each titler, there are two, is physically connected in before the corresponding VCR input. In order to add the titler video overlay, direct the video signal to the desired VCR/Titler destination. The video signal plus overlay may then be recorded and/or displayed. To display the output of the titler, direct the output of the VCR to the desired monitor. The titler will not work correctly with stereo video.

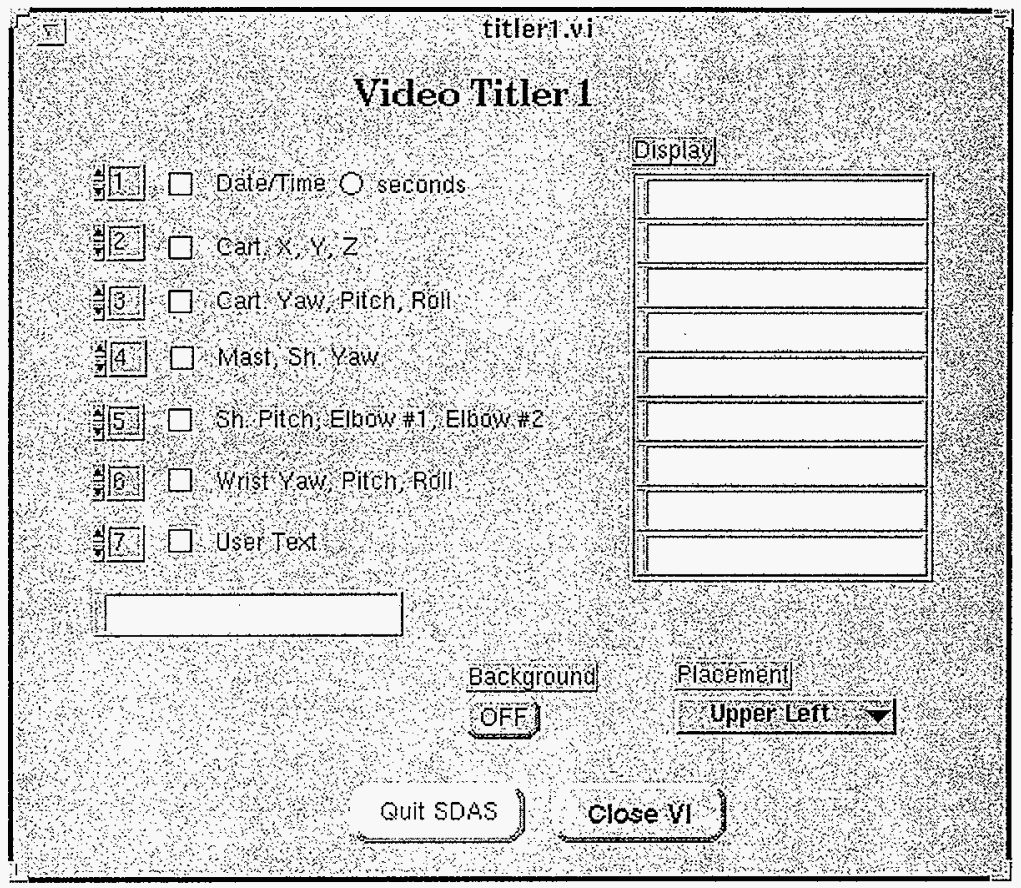

Figure 5 - Video Titler Control Panel

Simulated Display - This text display area shows approximately what the text will look like when displayed on the video screen.

Text Display Options - There are several text display options. Each option has a line number control and a check box. When the check box is marked with an "X", the option will be added to the Simulated display and will also appear on the video screen if it is currently being viewed. The line number control selects on which line of the display that option will appear. Line one is at the top and line nine is at the bottom. 
The control panel scans the list of options from top to bottom. If two or more options are checked, and are targeted for the same display line number, the last one in the list is what will be displayed.

Date/Time, Seconds - This option displays the date and time obtained from LabVIEW. This is not necessarily the same time you would get from the SDAS server. The Seconds button allows you to display seconds or not as you choose.

Cart. X, Y, Z - These are the X, Y, and Z Cartesian World coordinates obtained from the "Real" robot. The position of the "Simulated" robot is not available.

Cart. Yaw, Pitch, Roll - These are the Yaw, Pitch, and Roll Cartesian World coordinates obtained from the "Real" robot. The position of the "Simulated" robot is not available.

Mast, Sh. Yaw - These are the Vertical Positioning Mast, and Shoulder Yaw joint values obtained from the "Real" robot.

Sh. Pitch, Elbow \#1, Elbow \#2 - These are the Shoulder Pitch, Elbow \#1, and Elbow \#2 joint values obtained from the "Real" robot.

Wrist Yaw, Pitch, Roll - These are the Wrist Yaw, Wrist Pitch, and Wrist Roll joint values obtained from the "Real" robot.

User Text - This option displays whatever you type into the text field below, up to twenty characters.

\subsubsection{Other Controls}

Background Button - The titler displays text as a video overlay of white characters on whatever video is fed to it. If the video is generally dark, this works best, because the least video is obscured. However, sometimes the video is just to light and there is not enough contrast to read the text. Turn on the background button and a black background is placed behind the text. This makes the text easier to read, but obscures much more of the video.

Placement Selector - The titler overlays the text in a single large block of nine lines of twenty characters each line. The titler hardware allows you to specify the location of the upper left corner of this block by two arbitrary numbers between zero and sixty. Of these 3600 possible positions, we have selected four that place the corners of the block at corresponding corner of the screen. These are upper left, lower left, upper right, and lower right.

If you select one of the upper settings, place the text options near the top (line one). If you select one of the lower settings, place the text options near the bottom (line nine). If you select one of the left settings, the text options will be left justified for you. If you select one of the right settings, the text options will be right justified for you. Right justifying the text requires that spaces be placed at the front of the line. This is fine if the background is off, the spaces are transparent. However, if the background is on, the spaces become black and opaque, blocking whatever video is behind them. 
HNF-SD-TD-OMM-001, Rev. 1

Page 30

Close VI - Closes this Control Panel and returns control to the calling VI. If any-sub-vi's for this panel are still open, they must be closed before this panel can close.

Quit SDAS - Closes this and all other open panels except TopSDAS, disconnects the server and terminates VI execution. This button requires user verification. 


\subsubsection{Gripper End Effector Control Panel}

This panel is the control panel for Gripper End Effector as shown in Figure 6. It includes Position Control, Grip Force Control and Light Control as well as other controls.

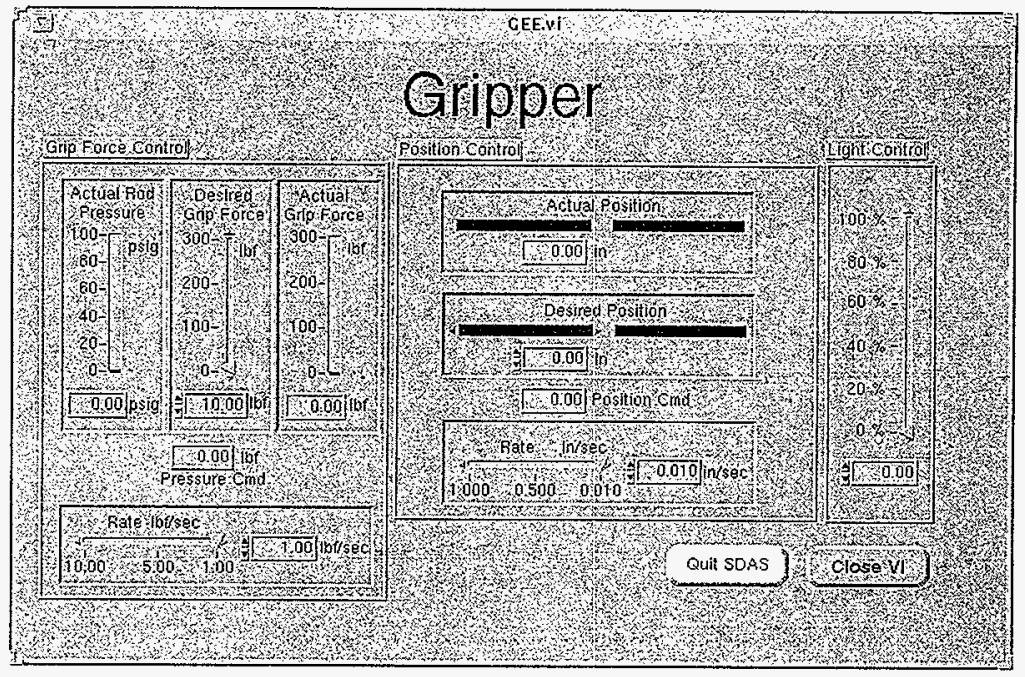

Figure 6 - Gripper End Effector Control Panel

\subsubsection{Position Control}

Actual Position Indicator-Indicates the actual gripper jaw opening in inches. This is a pair of sliding indicators that simulate the action of the gripper jaws and a numeric display of the actual opening in inches.

Desired Position Slider - Indicates and allows you to set the desired gripper jaw opening in inches. This is a pair of sliders that simulate the action of the gripper jaws and a numeric display of the desired opening in inches. The slider on the right and the numeric display may be set by the operator.

Rate Slider - The slider and numeric display controls the maximum rate at which the gripper jaws will open and close in inches per second. A larger number is faster. 
Position Cmd Display- This numeric display shows the actual desired opening value as constrained by the rate control. When the operator changes the desired opening, the display slowly follows until the values match.

\subsubsection{Grip Force Control}

Desired Grip Force Slider -This control is a slider and numeric display which establishes a set point for the desired maximum gripping force that the gripper applies to an object in poundsforce.

Actual Grip Force Indicator - This indicator is a slider and numeric display which displays the actual gripping force that the gripper applies to an object in pounds-force.

Actual Rod End Pressure Indicator- This indicator is a slider and numeric display which displays the actual pressure applied to the rod end of the air cylinder in pounds per square inch gage (psig).

Rate Slider - This slider and numeric display controls the maximum rate at which the gripping force is changed in response to the user changing the desired grip force slider in pounds-force per second. A larger number is faster.

Pressure Cmd Display - This numeric display shows the actual desired maximum gripping force value as constrained by the rate control. When the operator changes the desired gripping force, the display slowly follows until the values match.

\subsubsection{Light Control}

Light Slider- The slider controls the intensity of the light that illuminates the gripper in percent of full illumination. A larger number is greater intensity. The numeric display shows the control voltage $0-10$ volts.

\subsubsection{Other Controls}

Close VI-Closes this control panel and returns control to the calling control panel.

Quit SDAS - Closes this and all other open panels except TopSDAS, disconnects the server and terminates execution. This button requires user verification. 


\subsubsection{Optical Alignment Scope Control Panel}

This panel is the control panel for the Optical Alignment Scope as shown in Figure 7. It includes Light Control, Camera Control, Laser Control, Displacement Lasers as well as other controls.

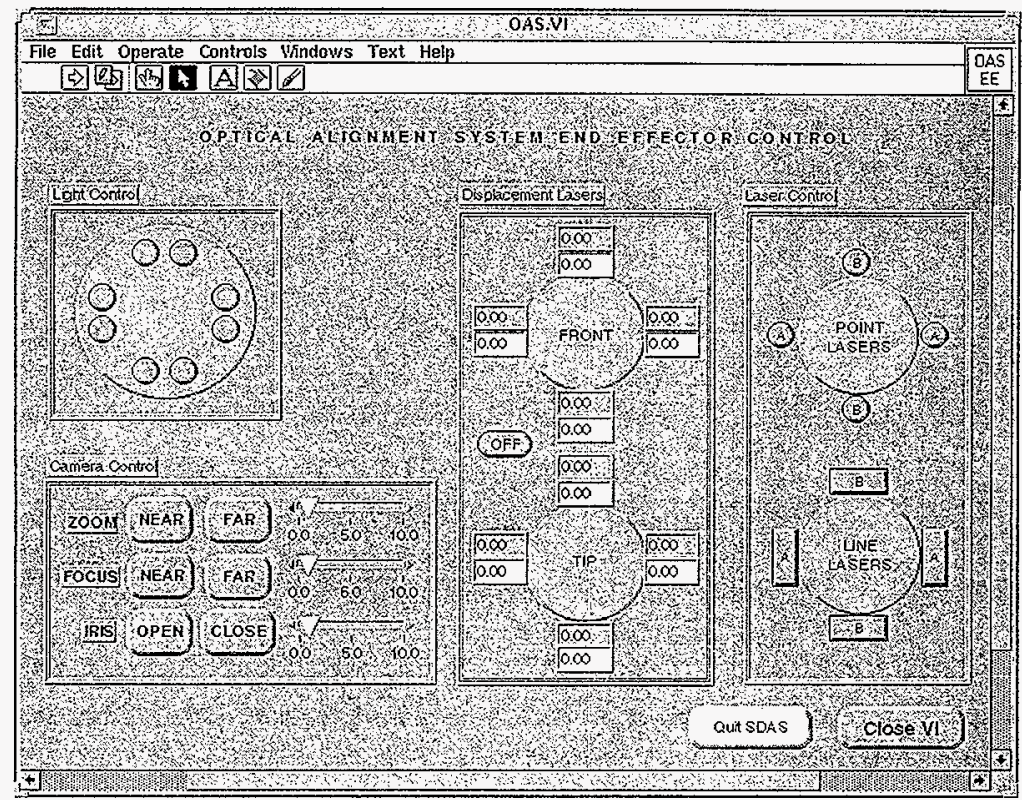

Figure 7 - Optical Alignment Scope Control Panel

\subsubsection{End Effector Orientation}

Many of the controls are shown symbolically located relative to each other and to the body of the end effector. The placement of each control is representative of the item it controls. The up/down and left/right orientation shown on the control panel is relative to the camera view.

\subsubsection{Light Control}

There are four control buttons in the Light Control section. Each button represents a pair of light bulbs on the front of the end effector. Each button will turn on the corresponding pair of lights. The buttons toggle, in for on, out for off. 


\subsubsection{Camera Control}

Zoom Out - Zooms the lens out while the control is depressed or until the lens is fully extended.

Zoom In-Zooms the lens in while the control is depressed or until the lens is fully retracted.

Zoom Slider - The slider controls the speed of the motor. A larger number is faster.

Focus Near - Focuses the lens closer while the control is depressed or until the lens reaches its near limit.

Focus Far - Focuses the lens farther away while the control is depressed or until the lens reaches its far limit.

Focus Slider - The slider controls the speed of the motor. A larger number is faster.

Iris Open - Increases/opens the iris while the control is depressed or until the iris is fully open.

Iris Close - Decreases/closes the iris while the control is depressed or until the iris is fully closed.

Iris Slider - The slider controls the speed of the motor. A larger number is faster.

\subsubsection{Laser Control}

Point Lasers $A$ - Each round button represents a point laser on the end effector. The lasers are controlled in pairs. Either of the buttons labeled "A" will turn on the corresponding pair of lasers. The buttons toggle, in for on, out for off.

Point Lasers $B$ - Each round button represents a point laser on the end effector. The lasers are controlled in pairs. Either of the buttons labeled "B" will turn on the corresponding pair of lasers. The buttons toggle, in for on, out for off.

Line Lasers $A$ - Each rectangular button represents a line laser on the end effector. The lasers are controlled in pairs. Either of the buttons labeled " $A$ " will turn on the corresponding pair of lasers. The buttons toggle, in for on, out for off.

Line Lasers $B$ - Each rectangular button represents a line laser on the end effector. The lasers are controlled in pairs. Either of the buttons labeled " $\mathrm{B}$ " will turn on the corresponding pair of lasers. The buttons toggle, in for on, out for off.

\subsubsection{Displacement Lasers}

On/Off - This button controls the power to eight displacement measurement laser on the end effector. The lasers are controlled all together. The button toggles, in for on, out for off. The label changes to show it's state. 
Page 35

Displacement Measurement Displays - These displays show the distance from the end effector to the nearest surface, which is normally the riser wall. Each display shows the reading from one of the eight displacement lasers in both inches and millimeters. The displays may be referenced as:

Top front

Bottom front

Left front

Right front
Top TIP

Bottom TIP

Left TIP

Right TIP

\subsubsection{Other Controls}

Close VI-Closes this Control Panel and returns control to the calling VI. If any-sub-vi's for this panel are still open, they must be closed before this panel can close.

Quit SDAS - Closes this and all other open panels except TopSDAS, disconnects the server and terminates VI execution. This button requires user verification. 


\subsubsection{High Resolution Stereo Video System Control Panel}

This panel is the control panel for the High Resolution Stereo Video System as shown in Figure 8, 9. It includes Lens Turret Control, Camera Selector, Closeup Control, and Light Control as well as other controls.

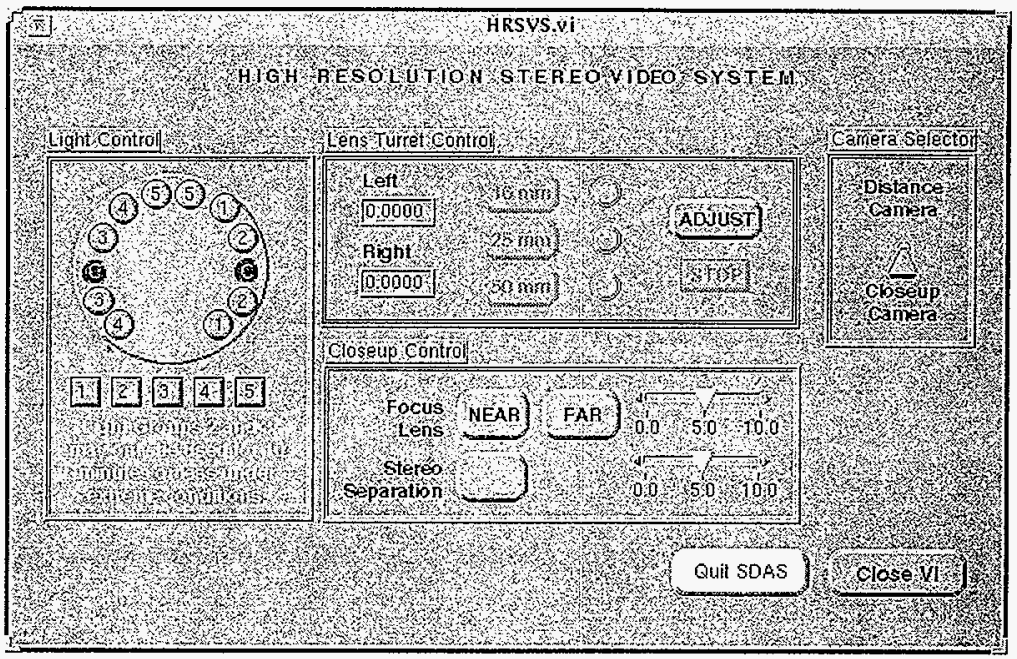

Figure 8 - High Resolution Stereo Video System Control Panel

\subsubsection{End Effector Orientation}

The light controls are shown symbolically located relative to each other and to the body of the end effector. The placement of each control is representative of the item it controls. The up/down and left/right orientation shown on the control panel is relative to the camera view.

\subsubsection{Light Control}

There are five control buttons in the Light Control section. Each button represents a pair of light bulbs on the front of the end effector. The buttons will turn on the corresponding pair of lights. The buttons toggle, in for on, out for off. 


\subsubsection{Camera Select}

Distance/Closeup - Switches between the distance camera pair and the closeup camera pair. When the distance camera is selected the Lens Turret Control and the Closeup Control areas are disabled and grayed.

\subsubsection{Lens Turret Control}

This section is no longer accurate. The function of the Lens Turret Control was modified to improve the control. All the buttons listed below, except the Adjust button have been disabled and will be removed in a future release.

$16 \mathrm{~mm} / 25 \mathrm{~mm} / 50 \mathrm{~mm}$ buttons - This group of three buttons select the lens pair used for the closeup cameras. The correct pair of lenses are rotated into position and centered at the nominal centered lens position.

Left/Right Position Indicators - Each position indicator gives feedback on the behavior of the lens turret. The position may vary from 0.00 to 1.00 .

Stop button - The stop button stops the lens turret motion regardless of whether the turret has reached the desired position.

Adjust button - The adjust button opens the lens turret fine position adjustment control panel.

\subsubsection{Closeup Control}

Focus Near - Focuses the closeup camera lens closer while the control is depressed or until the lens reaches its near limit.

Focus Far-Focuses the closeup camera lens farther away while the control is depressed or until the lens reaches its far limit.

Focus Slider - The slider controls the speed of the focus motor. A larger number is faster.

Stereo Separation Near - Change the stereo separation of the closeup cameras while the control is depressed. The cameras move alternately closer and farther apart. The function is cyclic, there is no limit.

Stereo Separation Slider - The slider controls the speed of the stereo separation motor. A larger number is faster.

\subsubsection{Other Controls}

Close VI - Closes this Control Panel and returns control to the calling VI. If any-sub-vi's for this panel are still open, they must be closed before this panel can close. 
HNF-SD-TD-OMM-001, Rev. 1

Page 38

Quit SDAS - Closes this and all other open panels except TopSDAS, disconnects the server and terminates VI execution. This button requires user verification. 


\subsubsection{High Resolution Stereo Video System \#2 Control Panel}

This panel is the control panel for the High Resolution Stereo Video System \#2 as shown in Figure 8, 9. It includes Lens Turret Control, Camera Selector, Closeup Control, and Light Control as well as other controls.

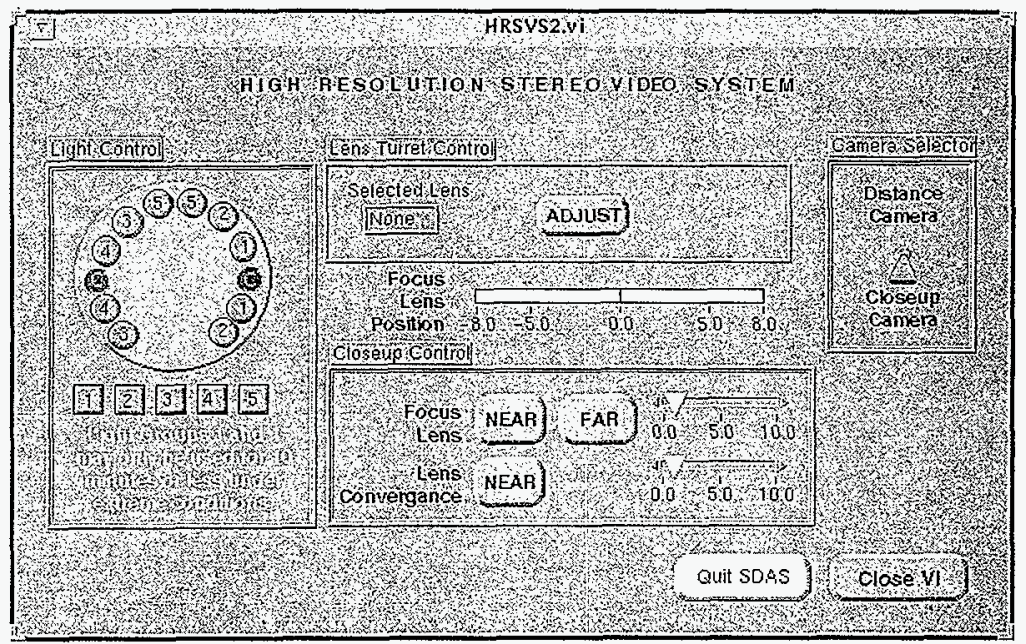

Figure 9 - High Resolution Stereo Video System Control Panel

\subsubsection{End Effector Orientation}

The light controls are shown symbolically located relative to each other and to the body of the end effector. The placement of each control is representative of the item it controls. The up/down and left/right orientation shown on the control panel is relative to the camera view.

\subsubsection{Light Control}

There are five control buttons in the Light Control section. Each button represents a pair of light bulbs on the front of the end effector. The buttons will turn on the corresponding pair of lights. The buttons toggle, in for on, out for off.

\subsubsection{Camera Select}

Distance/Closetip - Switches between the distance camera pair and the closeup camera pair. When the distance camera is selected the Lens Turret Control and the Closeup Control areas are disabled and grayed. 


\subsubsection{Lens Turret Control}

The Lens Turret Control section provides control of the stereo camera lens turrets..

Selected Lens Indicator - The selected lens indicator shows which of the stereo camera lens mounted on the lens turret is in position in front of the camera..

Adjust button - The adjust button opens the lens turret fine position adjustment control panel.

\subsubsection{Closeup Control}

Focus Near - Focuses the closeup camera lens closer while the control is depressed or until the lens reaches its near limit.

Focus Far-Focuses the closeup camera lens farther away while the control is depressed or until the lens reaches its far limit.

Focus Slider - The slider controls the speed of the focus motor. A larger number is faster.

Lens Convergence Near - Change the stereo separation of the closeup cameras while the control is depressed. The cameras move alternately closer and farther apart. The function is cyclic, there is no limit.

Lens Convergence Slider - The slider controls the speed of the stereo separation motor. A larger number is faster.

\subsubsection{Focus Lens Position}

Focus Lens Position Indicator - Designed to indicate the current position of the large focusing lens. This feature requires a hardware modification which is not currently installed.

\subsubsection{Other Controls}

Close VI - Closes this Control Panel and returns control to the calling VI. If any-sub-vi's for this panel are still open, they must be closed before this panel can close.

Quit SDAS - Closes this and all other open panels except TopSDAS, disconnects the server and terminates VI execution. This button requires user verification. 


\subsubsection{Lens Turret Position Adjustment Control Panel}

This is the Lens Turret Position Adjustment Control Panel as shown in Figure 10. It includes Turret Rotation Controls, and other controls. There is one for each of the two High Resolution Stereo Video Systems. The only difference is the number of lenses per turret that each control panel supports.

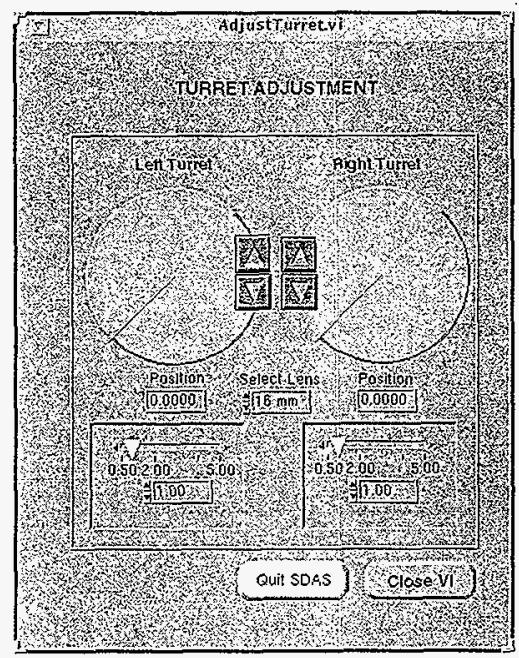

Figure 10 - Lens Turret Position

Adjustment Control Panel

\subsubsection{Turret Rotation Controls}

Matchmark Dial - Displays two positions as pointers on a dial. The green pointer indicates the position of the goal, and the blue pointer indicates the current position of the turret

Clockwise/Counterclockwise buttons - These four buttons cause the appropriate turret to rotate. The blue pointer moves to indicate the current position. The goal has been reached when the blue and green pointers line up.

Select Lens - Selects one of the lenses and sets the position of the green goal matchmark.

Position Indicator - gives a numerical indication of the turrets position.

Turret Speed Slider - Allows the operator to set the speed of the turret rotation motor. A larger number is faster 


\subsubsection{Other Controls}

Close VI - Closes this Control Panel and returns control to the calling VI. If any-sub-vi's for this panel are still open, they must be closed before this panel can close.

Quit SDAS - Closes this and all other open panels except TopSDAS, disconnects the server and terminates VI execution. This button requires user verification. 


\subsubsection{Robot Position Display Panel}

This is the display panel for the Robot Position Display Panel as shown in Figure 11, 31. It includes Cartesian Coordinates, Joint Coordinates, TipID Values, and other controls.

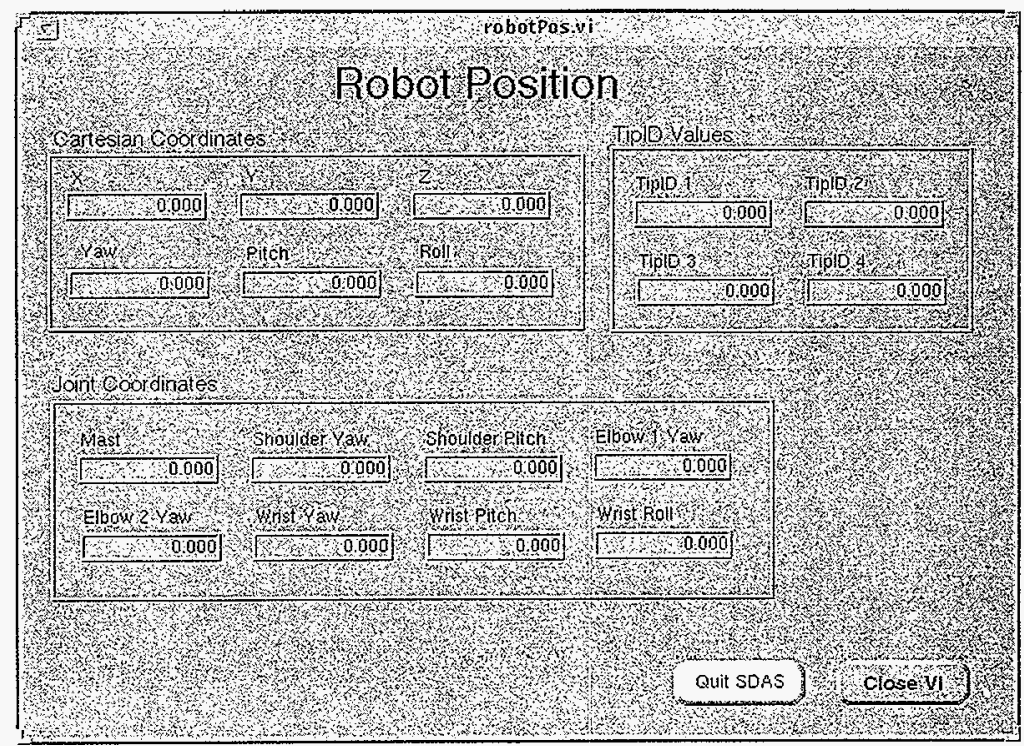

Figure 11 - Robot Position Display Panel

\subsubsection{Cartesian Coordinates}

The values displayed are the same values displayed on the LDUA Console as World coordinates.

$X$ - The translation along the $\mathrm{x}$ axis in inches.

$\mathrm{Y}-$ The translation along the $\mathrm{y}$ axis in inches.

$Z$ - The translation along the $z$ axis in inches.

Yaw - The rotation about the $\mathrm{x}$ axis in degrees.

Pitch - The rotation about the $y$ axis in degrees.

Roll - The rotation about the $z$ axis in degrees.

\subsubsection{Joint Coordinates}

The values displayed are the same values displayed on the LDUA Console.

Mast - Vertical Positioning Mast joint position in inches. 
HNF-SD-TD-OMM-001, Rev. 1

Page 44

Shoulder Yaw - joint angle in degrees.

Shoulder Pitch - joint angle in degrees.

Elbow $\# I$ - joint values angle in degrees.

Elbow \#2 - joint angle in degrees.

Wrist Yaw - joint angle in degrees.

Wrist Pitch - joint angle in degrees.

Wrist Roll - joint angle in degrees.

\subsubsection{Tool Interface Plate Identification Values}

TipID Values - These are the raw values supplied by the robot. They are voltage values supplied from the four voltage divider circuits in the Tool Interface Plate. Nominal values are 1-3 volts is a low or zero, 7-9.5 volts is a high or one. "TipID 1" is least significant, and "TipID 4" is most significant. Use the following table to convert the readings to an end effector number (0-15).

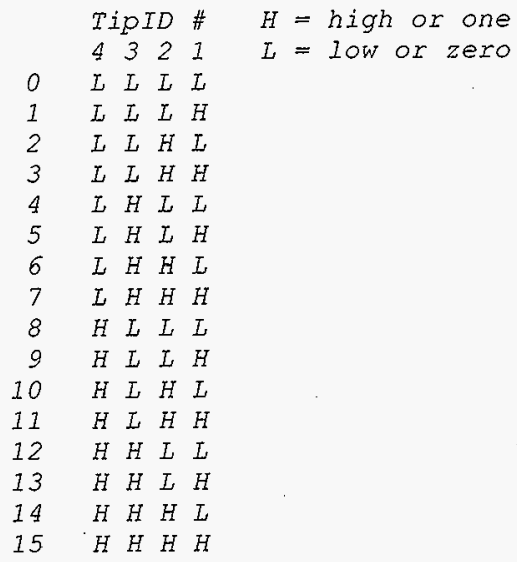

\subsubsection{Other Controls}

Close VI - Closes this Control Panel and returns control to the calling VI. If any-sub-vi's for this panel are still open, they must be closed before this panel can close.

Quit SDAS - Closes this and all other open panels except TopSDAS, disconnects the server and terminates VI execution. This button requires user verification. 


\subsubsection{Error Display Control Panel}

This panel is the Error Display Control Panel as shown in Figure 12. This panel displays SDAS server error messages, Opto-22 status, and internal SDAS server status. It is available from all of the top level control panels.

Here is a picture of the front panel.

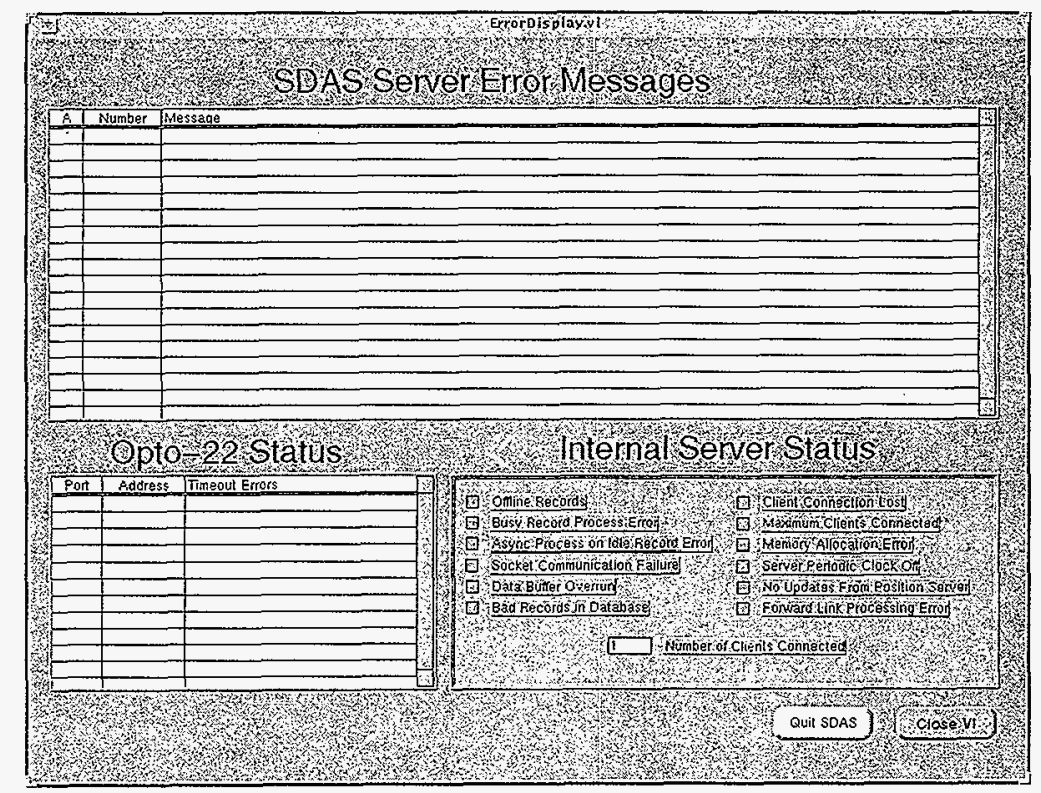

Figure 12 - Error Display Control Panel

\subsubsection{SDAS Server Error Messages Display}

Error List - This list displays all the errors posted by the SDAS server since the top level control panel was opened. Each error messages is stamped with time, date, process name and a sequence number. In addition, each message may be marked as acknowledged with an $\mathrm{A}$ in the first column. The error message list is loaded from a list mainatined by the top level control panel and is not updated until the control panel is closed and reopened. When it is opened, the first unacknowledged message is scrolled to the top. If there are no unacknowledged messages, oldest message is at the top of the list.

The time and date are generated by the SDAS server, which requests the current time from the SDAS Console once when it boots. After that, the clocks may drift apart. The SDAS Console clock is not synchronized to the line power and may also drift from wall clock time. The SDAS Console clock may be adjusted by the system administrator. 


\subsubsection{I/O Mux. Status Display}

Status List - This list displays a list of the Input/Output Multiplexor boards and their timeout error status. Each board is identified by the serial port to which it is attached and the board address. The timeout errors usually indicate that either the board has lost power, or the serial link has been interupted (i.e., a broken wire or loose connection). Here is a table listing the current configuration of the $1 / O$ multiplexor boards. All chassis are located in the At-Tank Instrument Enclosure.

\begin{tabular}{|l|l|l|l|l||}
\hline \hline Port & Address & \multicolumn{1}{|c|}{ System } & \multicolumn{1}{|c|}{$\begin{array}{c}\text { Chassis } \\
\text { Number }\end{array}$} & $\begin{array}{c}\text { Analog } \\
\text { Digital }\end{array}$ \\
\hline \hline 12 & 1 & Common Video End Effector Services & $6260 \mathrm{~A} 1$ & Digital \\
\hline 12 & 2 & Common Video End Effector Services & $6260 \mathrm{~A} 1$ & Analog \\
\hline 12 & 252 & Gripper End Effector & $4220 \mathrm{~A} 11$ & Analog \\
\hline 12 & 253 & Unused & $4220 \mathrm{~A} 11$ & Digital \\
\hline 12 & 254 & Radiation Monitoring & $4220 \mathrm{~A} 2$ & Analog \\
\hline 12 & 255 & Radiation Monitoring, Self-test, Water Decon & $4220 \mathrm{~A} 2$ & Digital \\
\hline
\end{tabular}

\subsubsection{Internal Server Status Indicators}

Offline Records - The indicator is red when there are offline records in the database, otherwise it is green. Offline records could be a temporary or a fatal condition, and indicates a condition that requires further operator action.

Bad Records In Database - The indicator is red when there are bad records in the database, otherwise it is green. This is a fatal condition that requires further action by the system cognizant engineer.

No Updates From Position Server - The indicator is green when the server is receiving position updates from the robot, otherwise it is red.

Database Processing Error Warning - This indicator is red when an attempt is made to process a record that is already being processed, otherwise it is green. An error on the error display will identify which channel is a problem.

Communication Error - The indicator is red when there are communication errors otherwise it is green. This is a fatal condition which requires the operator to reboot the SDAS server CPU. 
Maximum Clients Connected - The indicator is yellow when the maximum number of clients are connected to the server, otherwise it is green. Further connections will be refused when the maximum number of clients are connected. Since there are usually only a few connections made, this could indicate a problem.

Periodic Scheduler Off - This indicator is green when the servers scheduler is run, otherwise it is red. This is a fatal condition which requires the operator to reboot the SDAS server CPU.

Data Buffer Overrun - This indicator is red when there is a buffer overrun, otherwise it is green. When there is a buffer overrun data is lost on the LabVIEW side. This is not a fatal condition but indicates a performance problem. This problem should be logged and reported to the system cognizant engineer.

Number of Clients Connected - This indicator shows the number of clients currently connected to the server.

\subsubsection{Other Controls}

Close VI - Closes this control panel and returns control to the calling control panel. If other control panels were activated by this control panel, they must be closed before this one will close.

Quit SDAS - Closes this and all other open panels except TopSDAS, disconnects the server and terminates execution. This button requires user verification. 


\subsubsection{Supervisory Data Acquisition System Top Level Test Control Panel}

This panel is the main SDAS diagnostic control panel as shown in Figure 13. This panel controls and displays server connection, monitors system health, controls access to system diagnostic components.

Server connection is made automatically when TopTest is run. If a connection can not be made a message will be displayed and the control panel will be terminated. Once a connection is made the server is disconnected by selecting the Quit $S D A S$ button which requires user verification before disconnecting. The Quit SDAS button also terminates execution.

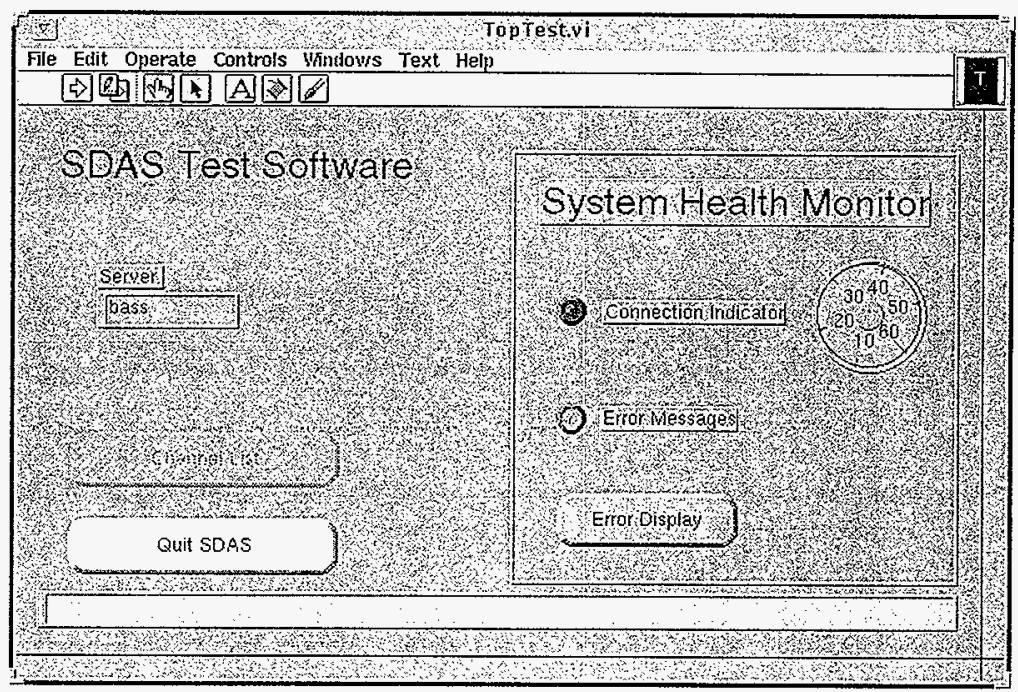

Figure 13 - Top Level Diagnostic Control Panel

\subsubsection{System Health Monitor}

The System Health Monitor section of the panel display includes the server connection, activity gauge, error message indicator, and error display button.

Connection Indicator - The indicator tells the user if a connection to the server has been made. This indicator is green when a connection exists and red when a connection does not exist. Lack of communication is a fatal condition. 
Activity Gauge - A gauge shows whether there is data flowing between the server and the control panel. This gauge will rotate when a connection exists and will stop when the control panel is no longer running or if there is condition inhibiting data flow between the server and the Control panel. Lack of data flow may indicate either a fatal condition or a performance problem.

Error Message Indicator - The indicator tells the user that server error message have been posted and may be observed on the Error Display Control Panel. If there are any unacknowledged error messages, this light is yellow.

Error Display Button - The button activates the Error Display Control Panel. Unacknowledged messages are acknowledged and the current error display is cleared.

Current Error Display - The display window at the bottom of the control panel shows the most recent unacknowledged message.

\subsubsection{System Components}

System components are accessed via control buttons. Controls that require a connection are greyed-out until a server connection is made. Each system component has its own display panel that is displayed by selecting the corresponding control button. System components that have not been integrated with SDAS will display an error message instead of the specified control panel. The controls are:

Server Name - The name of the SDAS server computer is hard coded into the other top level control panels. Since this control panel is intended for development, testing and diagnostics, the name of the SDAS server is in an editable text field. By changing this name, you may connect to any computer running the SDAS server software.

Channel List - Activates the Channel List control panel.

\subsubsection{Other Controls}

Quit SDAS - Closes any open panels except this panel, disconnects the server and terminates execution. This button requires user verification. 


\subsubsection{Channel List Control Panel}

This control panel as shown in Figure 14 is used to select channels from the SDAS channel database for display, modification, and reinitialization. This control panel is here for use in offnormal situations. It allows someone with a system programmer's level of knowledge to analyze the detailed behavior of the SDAS channel database. It is not to be used lightly, as arbitrary changes to the values stored will have unpredictable effects on the system.

Here is a picture of the front panel.

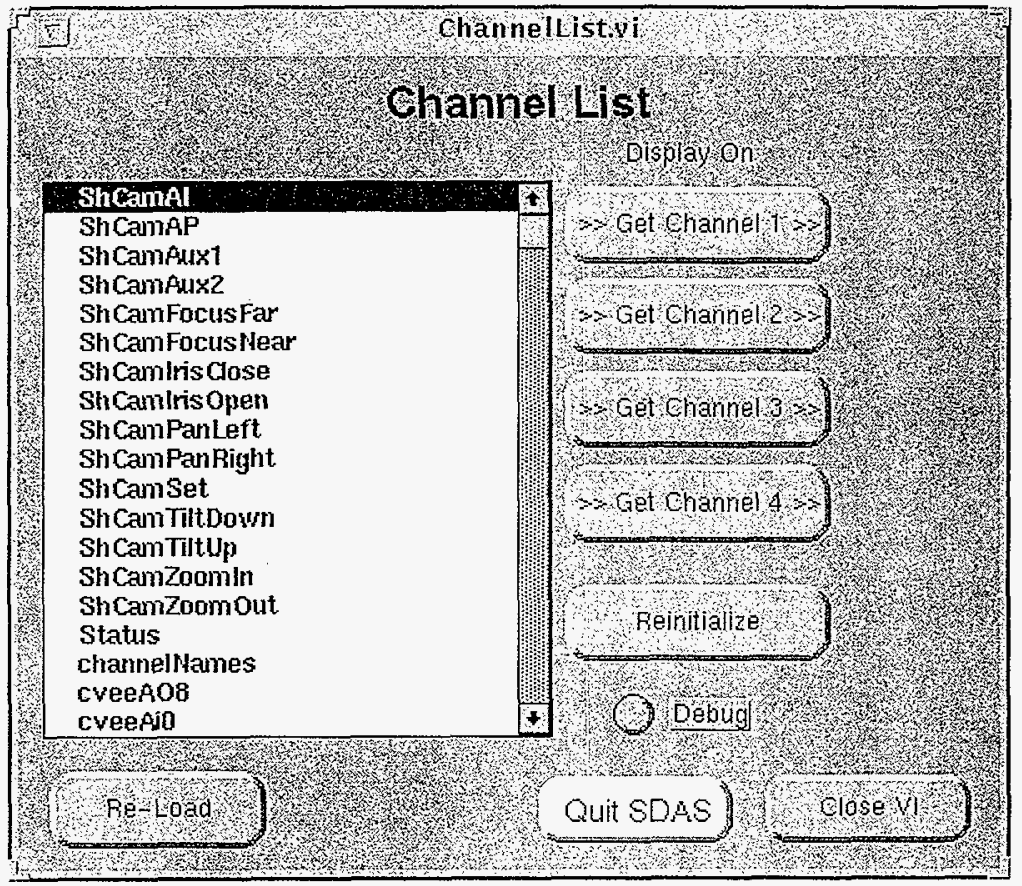

Figure 14 - Channel List Control Panel

\subsubsection{Channel Selection List}

Selection List - A list of valid channels is loaded from the database and displayed in the channel selection list box. A Loading indicator is displayed as the channel list is created. Loading automatically takes place when the Channel List panel is first opened. Further closing and opening of the Channel List panel does not trigger loading. Use the Re-Load button if you wish to reload the list. A column for symbols appears to the left of the channel names. A 0 symbol 
means the channel is offline and needs to be reinitialized. However, this is not currently implemented behavior. Modifications need to be made to the SDAS channel names driver to get this to work.

\subsubsection{Control Buttons}

Re-Load - The Re-Load control button allows the user to reload the channel list at any time. When this control is selected the loading activity is indicated by a red Loading label.

$>>$ Get Channel $\langle n\rangle>>\quad$ where $\langle n\rangle$ is $1,2,3$, or 4 . - After highlighting a channel from the channel list this control button activates a Channel Display control panel for the selected channel. A red check mark is displayed to the right of the button to indicate that a channel selection has been made.

Reinitialize - Reinitializes the selected channel by calling the driver reinitialization function if one is available. Reinitializing a record also schedules it for processing. The completion status is returned in a dialog and may be one of the following values:

Okay - the channel has been reinitialized.

Reinitialization not available - the driver for this channel does not support reinitialization.

Debug - The debug button sets a global debug flag. This has been used to activate checkpoint dialogue displays. Turning it on may result in unpredictable behavior. 1 believe that all instances of this flag have been removed, but I make no guarantees. Please leave it alone.

Close VI - Closes this control panel and returns control to the calling VI. If any sub-vi's for this panel are still open they must be closed before this panel can close.

Quit SDAS - Closes this and all other open panels except TopTest, disconnects the server and terminates VI execution. This button requires user verification. 
Page 52

\subsubsection{Channel Display Control Panel}

This panel as shown in Figure 15 is the channel display panel for displaying and modifying the values of selected channels in the channel database. It displays current channel information and controls processing of the selected channel.

Here is a picture of the front panel.

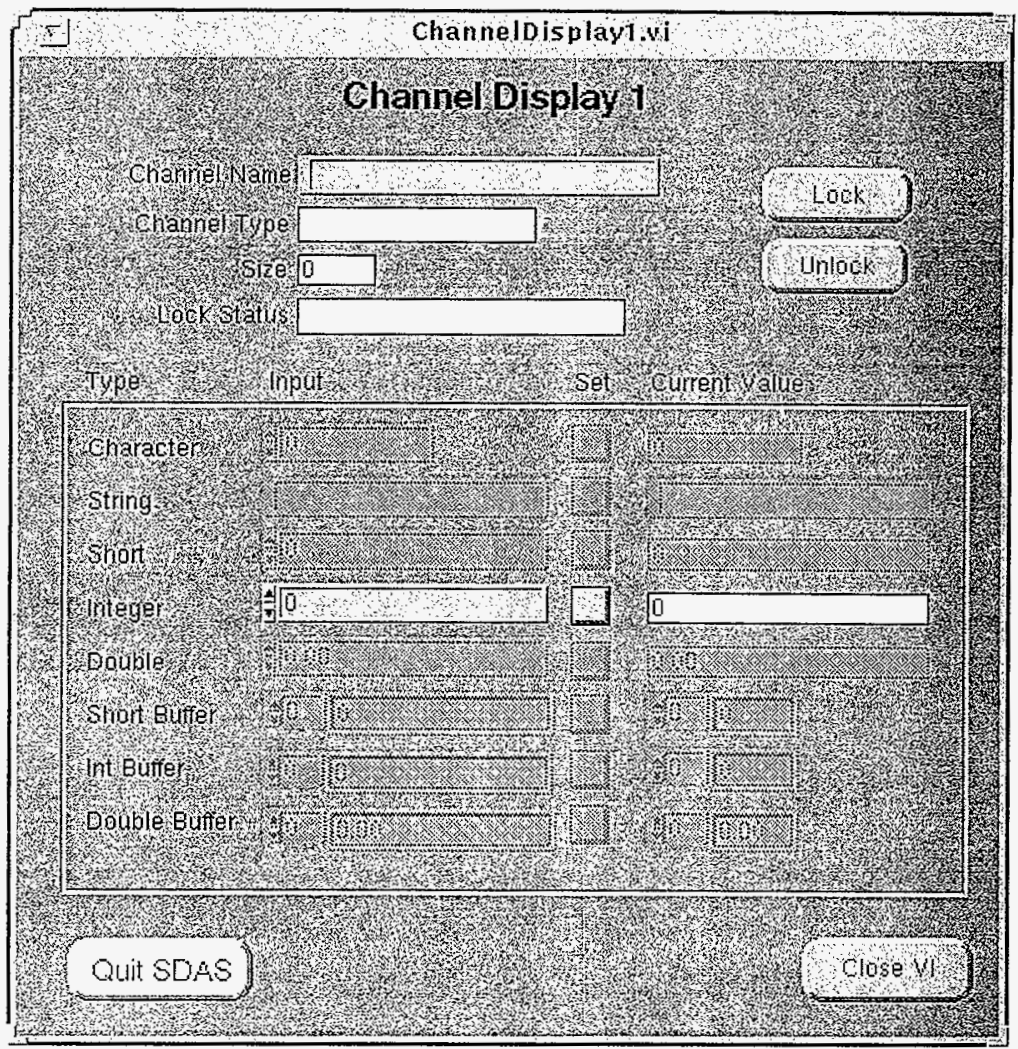

Figure 15 - Channel Display Control Panel 


\subsubsection{Channel Display}

Channel Name - Displays the current channel name.

Channel Type - Displays the valid data type for the selected channel.

Size - Displays the number of data elements for the selected channel. For non-buffered data types this value is always 1 , otherwise it is the number of buffer or array elements.

Lock Status - Displays the current channel lock status.

Unlocked

Locked (Another User)

Locked (Self)

Lock Error write access is enabled for all clients.

write access is denied. Another client has locked the channel.

write access is denied to others. This client has write access.

An error was encountered while trying to lock the channel. The channel was already locked by another user.

\subsubsection{Channel Value}

This section includes the following information for all possible data types. The controls and indicators are enabled for the current data type only.

Type - Identifies the data types. Supported data types are: Character - a signed or unsigned byte

String - an array of signed or unsigned bytes

Short - a signed or unsigned 2-byte integer

Integer - a signed or unsigned 4-byte integer

Double - an 8-byte double precision floating point

Short Buffer - an array of signed or unsigned 2-byte integers

Int Buffer - an array of signed or unsigned 4-byte integers

Double Buffer - an array of 8-byte double precision floating points

Input - User specified data to be sent to the selected channel.

Set - Writes the input data value to the selected channel.

Current Value - Displays the current value for the selected channel. 
HNF-SD-TD-OMM-001, Rev. 1

Page 54

\subsubsection{Other Controls}

Lock - Disables write access to the selected channel from any client other than this one.

Unlock - Enables write access to the selected channel by all clients.

Close VI - Closes this control panel and returns control to the calling VI. If any sub-vi's for this panel are still open they must be closed before this panel can close.

Quit SDAS - Closes this and all other open panels except TopTest, disconnects the server and terminates VI execution. This button requires user verification.

\subsubsection{Supervisory Data Acquisition System Guest Top Level Control Panel}

This panel is the guest SDAS control panel as shown in Figure 16. This panel controls and displays server connection, monitors system health, and controls access to guest video system components.

Server connection is made automatically when GuestSDAS is run. If a connection can not be made a message will be displayed and the control panel will be terminated. Once a connection is made the server is disconnected by selecting the Quit SDAS button which requires user verification before disconnecting. The Quit SDAS button also terminates execution.

Here is a picture of the front panel.

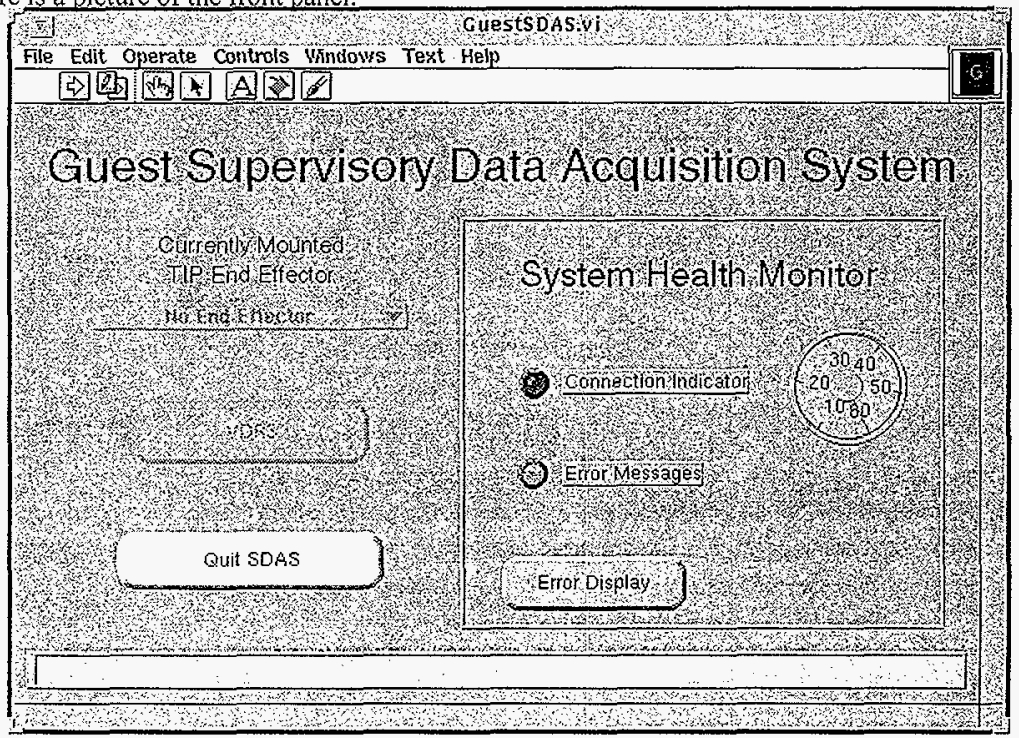

Figure 16 - Guest Top Level Control Panel 
HNF-SD-TD-OMM-001, Rev. 1

Page 55

\section{3,4.16.1 System Health Monitor}

The System Health Monitor section of the panel display includes the server connection, activity gauge, system error indicator, and error display button.

Connection Indicator - The indicator telis the user if a connection to the server has been made. This indicator is green when a connection exists and red when a connection does not exist. Lack of communication is a fatal condition.

Activity Gange - A gauge shows whether there is data flowing between the server and the control panel. This gauge will rotate when a connection exists and will stop when the control panel is no longer running or if there is condition inhibiting data flow between the server and the Control panel. Lack of data flow may indicate either a fatal condition or a performance problem. System Error Indicator - The indicator tells the user that errors have occurred and may be observed on the Error Display Control Panel.

Error Message Indicator - The indicator tells the user that server error message have been posted and may be observed on the Error Display Control Panel. If there are any unacknowledged error messages, this light is yellow.

Error Display Button - The button activates the Error Display Control Panel. Unacknowledged messages are acknowledged and the current error display is cleared.

Current Error Display - The display window at the bottom of the control panel shows the most recent unacknowledged message.

\subsubsection{System Components}

System components are accessed via control buttons. Controls that require a connection are greyed-out until a server connection is made. Each system component has its own display panel that is displayed by selecting the corresponding control button. System components that have not been integrated with SDAS will display an error message instead of the specified control panel. The controls are:

Currently Mounted TIP End Effector - The currently mounted TIP end effector may be either determined from information gotten from the robot or selected from a pull-down menu. This control is grayed out and the label changes automatically with changes of the end effector, if robot position information is available.

Once the currently mounted TIP end effector has been selected a control button for that end effector is enabled. This control button activates a control panel for the selected end effector.

The mounted TIP end effector may supply a video source to the Video Display and Recording System. The settings on both the TopSDAS control panel and the VDRS control panel must be compatible for correct operation. Changing one might require changing the other as not all end 
HNF-SD-TD-OMM-001, Rev. 1

Page 56

effector types are compatible with all video destinations. The user will be alerted when a setting has changed unless both the previous and new end effector do not supply a video source.

VDRS - Activates the Guest Video Display panel.

\subsubsection{Other Controls}

Quit SDAS - Closes any open panels except this panel, disconnects the server and terminates execution. This button requires user verification. 


\subsubsection{Guest Video Display Control Panel}

This VI is the guest Video Display control panel as shown in Figure 17. The user can select video sources for the available guest video destinations, which are video monitors five and six, and stereo monitor two. There is a companion VI that controls the main video settings. See the Video Display and Recording Control Panel.

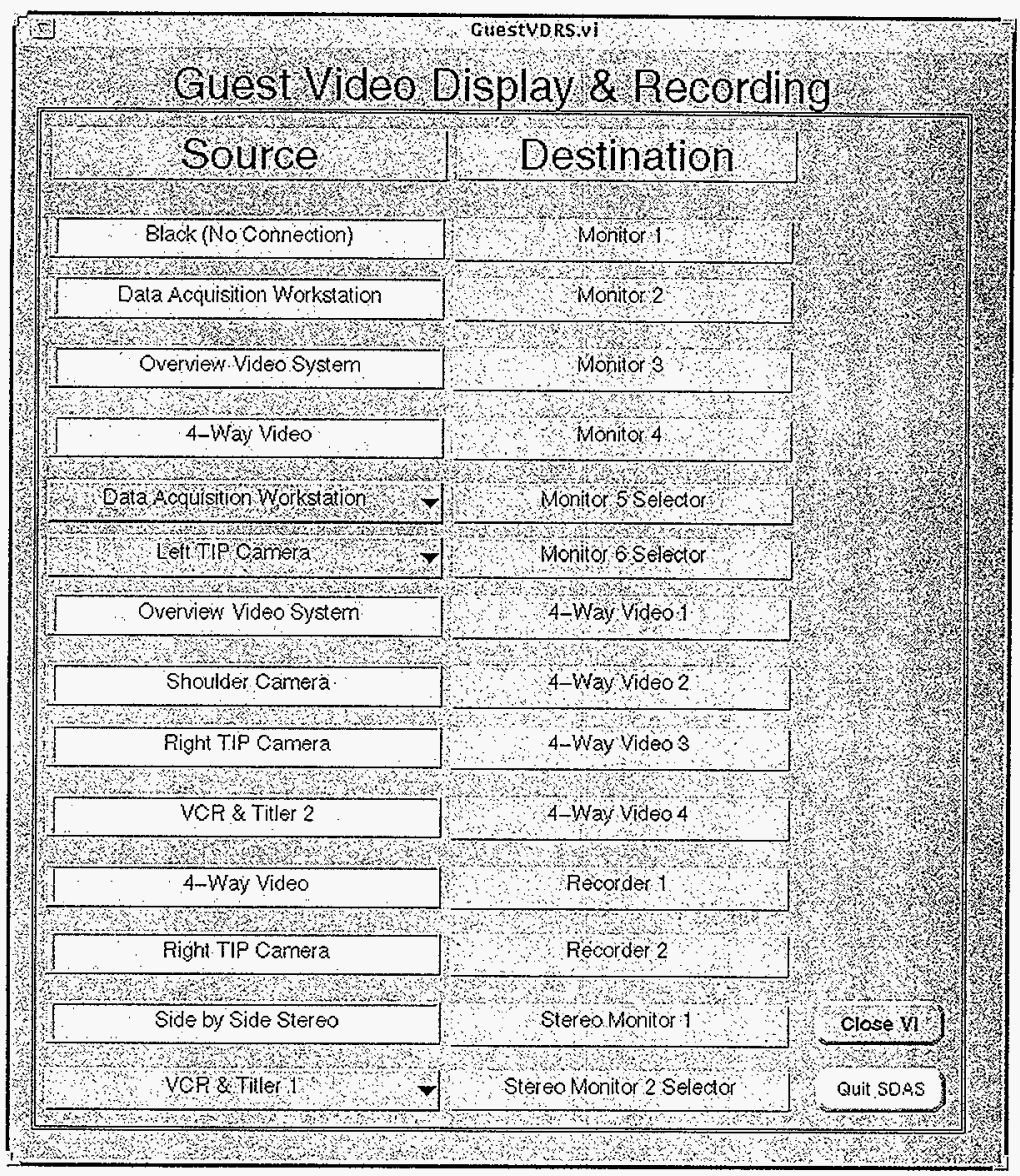

Figure 17 - Guest Video Display Control Panel 
Each destination consists of a label and either a pull-down menu or a simple display of possible source settings for that destination. The menus are unique to each destination or destination type, allowing connections to only those sources that are sensible.

The Mounted TIP End Effector, Left TIP Camera, and Right TIP Camera sources refer to the current end effector mounted on the system. The mounted TIP End Effector is set from the GuestSDAS control panel. These settings must be compatible for correct operation. Changing one might require changing the other as not all end effector types are compatible with all destinations. The user will be warned when a change does not meet the minimum requirements for operation. The minimum requirements are defined below for each source/destination combination.

There are six video monitors. The display source for the fifth and sixth monitors is controlled from this panel using pull-down menus. The pull-down menus for these monitors are identical. The display source for the first four monitors will be shown on this panel but can not be controlled. The possible sources are:

\section{4-Way Video}

Black (no connection)

Data Acquisition Workstation

Left Tip Camera-Minimum requirements are that the end effector has a left camera.

Mounted TIP End Effector - Minimum requirements are that the end effector has s-video. Operations Workstation

Right Tip Camera - Minimum requirements are that the end effector has a right camera.

Shoulder Camera

Side by Side Stereo

Overview Video

Operations Overview Video

VCR \& Titler 1

VCR \& Titler 2

There are two Stereo Monitors. The display source for the second monitor is controlled from this panel using pull-down menus. The display source for the first monitor will be shown on this panel but can not be controlled. Some sources require that the signal first pass through a stereo converter before being sent to the Stereo Monitor. This conversion is controlled by the main operator. If the main operator does not have this conversion setup, this display panel can not affect it. The possible sources are:

Black (no connection)

Stereo Video Converter

VCR \& Titler 1

VCR \& Titler 2

\subsubsection{Other Control Buttons}

Close VI - Closes this control panel and returns control to the calling VI. If any sub-vi's for this panel are still open they must be closed before this panel can close. 
Quit SDAS - Closes this and all other open panels except TopSDAS, disconnects the server and terminates VI execution. This button requires user verification.

\subsection{SCS EQUIPMENT OPERATION}

\subsubsection{World Model Overview}

The world model software for the Light Duty Utility Arm (LDUA) has been developed to give a three dimensional representation of the robot, tank, and all equipment that may be necessary for a given task. The software is based on IGRIP, version 2.3 or above, running on a Silicon Graphics computer. The intent was to develop a package such that an operator could use the software without having experience with Silicon Graphics computers, or IGRIP.

The software has been developed by customizing IGRIP with macros and user buttons for functions that are necessary to render the LDUA, tanks, and any hardware within the tank. IGRIP is used as the basic platform to render the robot and all equipment in three dimensions, provide near miss and collision checking, and provide the basic interface. The user customized buttons provide specific functionality that is required for the LDUA.

\subsubsection{World Model Installation}

This section outlines how to install the world model software on a computer to be used with the LDUA. This procedure assumes that IGRIP has already been installed on the computer, and that it is functioning properly. It also assumes that the installer has experience with IGRIP and SGI computers.

1. Unpack the files into the oper account home directory using the command:

zcat WorldModel.revision.tar.Z I tar xvf -

This will create a directory named WorldModel.

2. Create a link for the IGRIP user button configuration file as follows:

In WorldModel/button_config. igbutfig (For IGRIP installations)

In WorldModel/button_config . telbutfig (For TELEGRIP installations)

3. Edit the "World_Model" script file, located in the <home $>$ /WorldModel directory, and change the directories to reflect the IGRIP installation directory and the operator directory. Note:, the LDUA.ig file is a configuration file located in the $<$ home $>$ /WorldModel directory.

4. Change directories to the $<$ home $>/$ WorldModel/macros directory and edit all *.gsl files changing all references to the $<$ home $>$ /WorldModel directory. In each file, change the "LIBRARY" constant variable to reflect the operator's account directory (to $<$ home $>$ (WorldModel).

5. Edit the LDUA.ig file. At the top of this file is a number of directories that need to be changed to reflect the home directory of the operator account. All lines that need to be changed have the '\$LIB' string in them. Note: These are the same as the '.igpthfig' file referenced in the 
HNF-SD-TD-OMM-001, Rev. 1

Page 60

IGRIP user manuals. This file replaces the '.igpthfig' and the '.igenvfig' files, and therefore IGRIP will not read those files when running the World_Model script.

6. Drag the World_Model script file from a file window onto the upper right hand corner of the Silicon Graphics desktop (screen) and label it "World Model".

7. Double click on the "World Model" icon and start the testing procedure.

\subsubsection{World Model Operation}

This section assumes that the Spar SGCS has already been installed and is running when the operator logs in. The Hanford LDUA installation starts the SGCS at system startup and it runs continuously, provided that the Arm Level Controller was available during system startup. See the applicable startup procedure.

\subsubsection{Starting the World Model}

To start the world model software, the operator needs to select the icon labeled "World Model" that is located in the upper right hand corner of the Silicon Graphics desktop (screen) after the operator has logged onto the computer.

After IGRIP has loaded into memory, and loaded the LDUA model, it will display the LDUA. model at the center of the screen. IGRIP's operator interface will present the operator with buttons on the top, bottom, and right side as seen below in Figure 19. The operator should select the "User" button in the top menu bar. This should highlight the "User" button and change the right menu choices, and display the "Choose Tank", "Locate LDUA" and other LDUA specific buttons in the right side menu area. If the "User" button is highlighted, and the "Choose Tank" and "Locate LDUA" buttons don't appear in the right menu area, then the operator may have to select the LDUA button located near the top right of the screen. This should bring up the user menu. Once setup properly, All buttons pictured in figure 18 will be available to the operator. 
Page 61
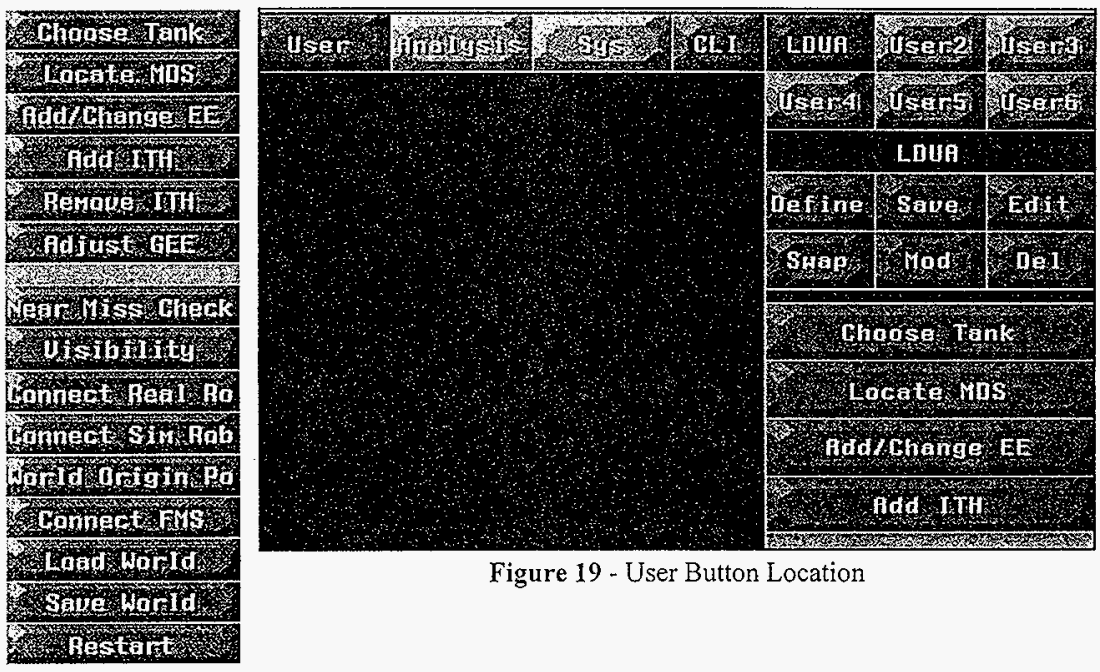

Figure 19 - User Button Location

Figure 18 - World

Model User Buttons

\subsubsection{Choosing the Tank Model}

To choose the desired tank for the LDUA deployment, the operator must first press the "Choose Tank" button in the right menu area. This will bring up a menu box seen in Figure 20. To choose the tank, the operator must select the desired tank from the menu with the mouse using the left mouse button. After a short wait, this should bring a tank into view. To choose another tank, the operator can reselect the "Choose Tank" button, and run the macro again and it will replace the existing tank with the new one that is selected. Please note that all In-Tank Hardware in the display will be removed when a new tank is chosen.

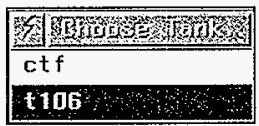

Figure 20 - Choose

Tank Pop-up

\subsubsection{Orienting the LDUA to the World Model}

Orienting the LDUA/MDS requires that the operator knows the riser that the LDUA is installed in, the orientation of the LDUA with respect to the world, and the height from the top of the riser to the bottom of the LDUA TIP. To locate the LDUAMMDS, the operator must select the "Locate MDS" button located in the right menu area. This will bring up another menu box as seen in 
Figure 21. This has five menu items that the operator must complete to position the MDS/LDUA. To select the riser, the operator must select the name at the top of the right column. Each click of the mouse button will scroll through to the next riser for each tank. When the correct riser name is in the window, then it has been selected. To change the yaw, pitch, azimuth, and height of the LDUA, the operator must first select the number beside the desired parameter, and type in the correct value. Once all values have been set, the operator can select the "Done" button at the top right of the menu window. This will place the LDUA above the proper riser, and in the proper orientation. The operator should visually check to make sure that the configuration of the robot appears correct on the screen. If the location is incorrect, the operator may start over by selecting the "Locate MDS" button again.

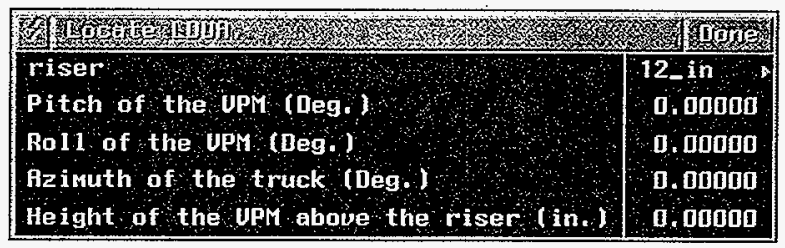

Figure 21 - Locate LDUA Pop-up

\subsubsection{Adding or Changing the End Effector}

The next step is to choose the end effector for to be used for the LDUA deployment. The operator should select the "Add/Change EE" Button to bring up the End Effector selection menu shown in Figure 22. To choose the end effector the operator must select the desired unit. If the desired end effector is not listed in the menu, then the generic end effector may be selected, which consists of a cylinder that is the maximum allowable size for an end effector. This will allow all collision detection algorithms to work properly. At any time during the deployment, the operator may select a different end effector with this button.

To remove the end effector, select "none" from the selection menu.

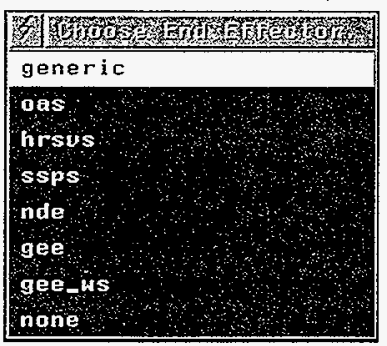

Figure 22 - Choose End Effector Pop-up 


\subsubsection{Adding In Tank Hardware (ITH)}

To add In Tank Hardware (ITH) the operator should select the "Add ITH" button located in the right menu area to bring up the Add ITH menu as shown in Figure 23. The next window that comes up is one that enables the operator to select the riser that contains the ITH as shown in Figure 24. If the desired ITH is not present in the first menu, a generic option is available. If a generic ITH type is chosen, another menu will come up to prompt the operator to give the dimensions of the ITH. The operator will input the diameter and length of the ITH for modeling purposes. The Generic ITH is simply a cylinder of the given diameter and length which allows for the worst possible constraints for the collision avoidance software routines. The Add ITH button may be chosen as many times as necessary to place all know ITH devices in the tank setup.

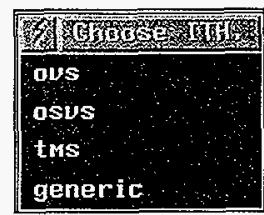

Figure 23 -Choose ITH Pop-up

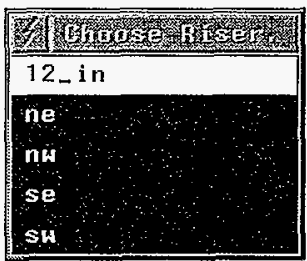

Figure 24 - Choose Riser Pop-up

\subsubsection{Removing or Changing In Tank Hardware}

To remove an In Tank Hardware (ITH) Device, the operator should select the "Remove ITH" button located in the right menu area to bring up the Delete ITH menu as in figure 25 . This menu shows all the ITH Devices installed in this tank. In each menu choice, the first word is the name of the ITH Device. The second word in each menu choice is the name of the riser in which the ITH Device is located. Select the one device to be deleted to remove it from the tank setup. To change an ITH Device, first remove it from the tank setup, then add the correct information with the "Add ITH" button.

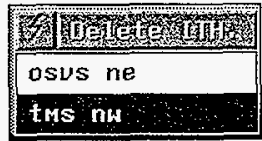

Figure 25 - Remove

In Tank Hardware 
HNF-SD-TD-OMM-001, Rev. 1

Page 64

\subsubsection{Adjust Gripper End Effector}

Occasionally, when the Gripper End Effector (GEE) is on the LDUA, the operator may want to simulate the opening and closing of the GEE in the model. To do this, select the "Adjust GEE" button in the right menu area. This will bring up the GEE box, as shown in figure 26 , which will allow the operator to select the distance between the jaws of the gripper. To simulate the GEE correctly, input the value from the SDAS interface in the dialog box to open the simulated GEE jaws. This will work for the GEE, and the GEE with Waste Probe (gee_ws).

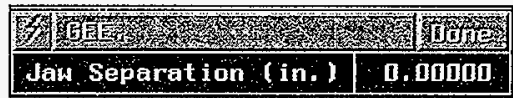

Figure 26 - Adjust Gripper Jaw Seperation

\subsubsection{Using Collision Avoidance}

Once the tank has been set up with the proper end effector and ITH devices, then the operator may turn on the collision avoidance algorithms. In this mode, the software will highlight impending collisions or actual collisions as predicted by the computer model. This is intended as an aid to the operator to assist him in avoiding collisions in the tank. Note: Neither the World Model software nor the LDUA control software will take any automatic action - the operator is fully responsible for the safe operation of the equipment.

To activate collision avoidance select the "Near Miss Check" Button to bring up the Near Miss Check Menu as seen in Figure 27. By selecting "ON" or "OFF" next to the "Near Miss Checks" prompt, the operator can turn the near miss checks on or off. The "Near Miss Distance" allows the operator to change the minimum distance between objects for the software. Selecting "Done" sets the desired values into the software.

Once the near miss checks have be activated, the tank setup will change color in the event of a collision or a near miss. When a near miss situation exists, the tank and LDUA in the tank setup will change color to blue, and the parts that are too close will change color to yellow. If a collision were to occur, the parts that are colliding will turn red.

A near miss situation will occur while the LDUA is stowed, or partially deployed. This is due to the fact that the LDUA joints are near the riser and walls of the tank setup until it has been deployed the minimum distance below the roof of the tank. This means that the near miss checks should not be turned on until the LDUA is deployed such that the inner tube clears the roof of the tank.

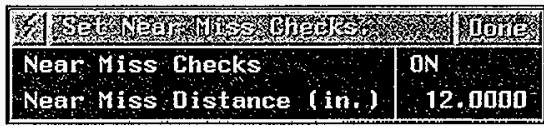

Figure 27 - Set Near Miss Checks Pop-up 


\subsubsection{Setting the Visibility of the Robot}

Occasionally, the operator may want to make the LDUA or the Simulated LDUA invisible or visible during the operation. To achieve this, select the "Visibility" button in the right menu area. This will bring up the message window as in figure 28 . Select yes to make each simulation visible, and no to make them invisible.

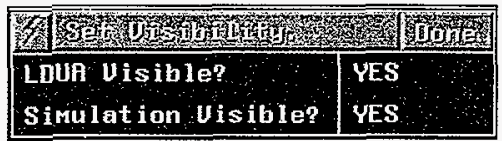

Figure 28 - Set Visibility

\subsubsection{Connecting the Real Robot}

When using the World Model as a simulation tool, the operator will want to connect the simulation to the actual robot so that the simulated robot will mimic the actions of the actual robot. To do this, select the "Connect Real Robot" button in the right menu area, which will bring up the dialog box as in figure 29. By clicking with the left mouse button on the word Connect, you can connect or disconnect the World Model robot from the real robot.

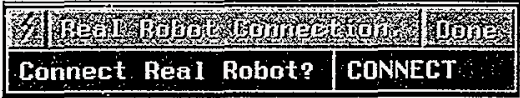

Figure 29 - Real Robot Connection

\subsubsection{Connecting the Simulated Robot}

The SPAR controller allows the operator to preview a move using a simulated robot, which is controlled by the same hardware that controls the actual robot. To connect the Simulation robot in the World Model to the Simulated robot in the SPAR hardware, select the "Connect Sim Robot" button in the right menu area. This will bring up a similar dialog box to the one used above, pictured below in figure 30. Again, select connect or disconnect to allow the World Model Simulation robot to the Hardware.

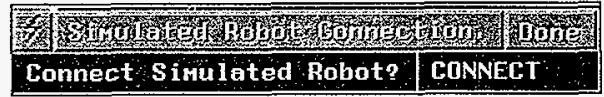

Figure 30 - Simulated Robot Connection 


\subsubsection{Determining Robot Position in World Coordinates}

For some end effectors, the origin of the robot needs to be known with respect to the origin of the world model. This can be easily retrieved from the world model by using the "World Origin Pos" button in the right menu area. This will display the location of the LDUA with respect to the world model origin, in IGRIP's standard $x, y, z$, roll, pitch, yaw notation. This button will display a box such as figure 11,31 below.

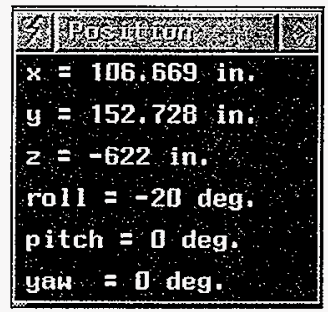

Figure 31 - Robot Position

\subsubsection{Displaying the Force Moment Sensor Data}

In order to determine the forces acting on the LDUA during deployment, the operator may want to display the data from the Force Moment Sensor (FMS). To do this, the operator must select the "Connect FMS" button in the right menu area which will bring up a dialog box as pictured in figure 32. To connect the FMS sensor, the operator must make sure the dialog box displays "CONNECT", and press the done button. When connected, the World Model Software will display an arrow, which displays the forces acting on the robot. Note: The FMS sensor is not a calibrated instrument. The readouts are for information only and do not define a safe operating envelope.

To turn off the display of FMS data, select the "Connect FMS" button, click on the word "CONNECT" until it displays "DISCONNECT", and press the done button.

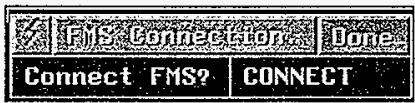

Figure 32 - Force Moment Sensor

Connection

\subsubsection{Loading Saved Tank Setups}

Whenever you setup a tank model, the software maintains a record of the current configuration. This configuration may be restored the next time you start the system by selecting the "Restart" button. However, since you may want more than one saved tank setup, it is possible for an 
operator to save a certain configuration of tank, ITH, end effector, and position data in a file for later use. Once data is saved, it can be reloaded at any time. To do this, select the "Load World" button in the right menu area, which will bring up a dialog window as in figure 33 . To load a saved world, select the desired file, and press done.

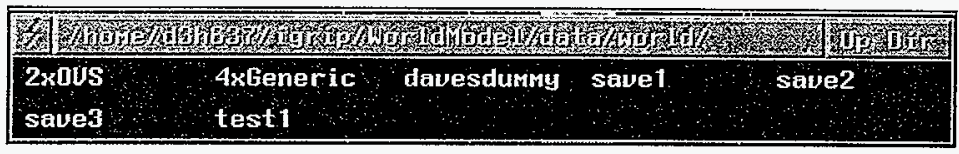

Figure 33 - Loading Saved Tank Setups

\subsubsection{Saving Tank Setups}

During a deployment, it is possible for an operator to save a certain configuration of tank, ITH, end effector, and position data in a file for later use. To save the current deployment information to a file to be retrieved later, press the "Save World" button in the right menu area. This will bring up two boxes as pictured in figure 34. The "Message Window" box lets the operator know where the file is being saved to, the "File Name" box will ask for a filename and a path name. The path name will be added to the Don't enter a path name unless you are familiar with the windowing system. To save the file type a name into the File Name space in the window, and the data will be save to that file, in the same directory that the "Load World" button loads from.

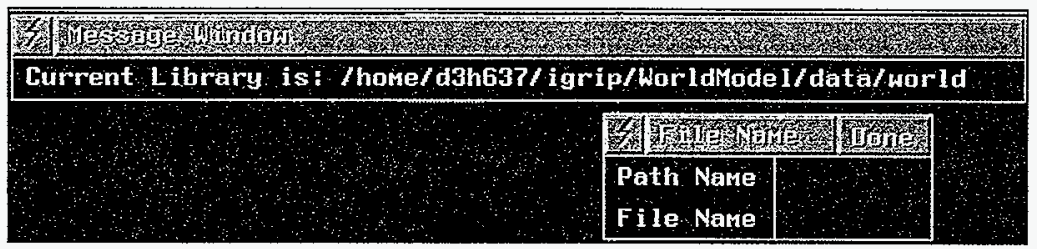

Figure 34 - Saving Tank Setups

\subsubsection{Restoring Previous Settings}

Whenever you setup a tank model, the software maintains a record of the current configuration. This configuration may be restored the next time you start the system by selecting the "Restart" button. In the event that the storage file is somehow corrupted, this function may fail, and the model will not appear correctly. If this happens, the operator must load the tank setup from a saved file or set up the tank from the scratch. 


\subsubsection{Modifying the World Model}

New items must be added to the computer model as they become available so that they can be included in the world model software. These items include tanks, end effectors, and in tank hardware. To include these items, a developer must add data files, and append data files for the world model, as well as developing IGRIP models of the items to be used. In this section, a description of the process of adding each of these items is included, and a more detailed description of the data files is included in section 3.5 .5 along with sample data files.

Modifying the World Model Software constitutes a change to the software configuration. Therefore all changes must follow the applicable change control procedures.

\subsubsection{Creating New Tank Models}

To create new tanks for the world model, you must follow a few conventions so that the macros work correctly. These conventions are as follows:

1. The tank model that is created must be saved as a device in the 'devices/tanks' directory under the main directory structure. The file name used should be the name of the tank (i.e. 't106'). This device should include the tank parts, along with a separate part for each riser. The riser parts should have their origin in the center of the pipe, and where the riser meets the inside surface of the dome. Note: the 106 model was created using drawing number BPF 73550 which covers all of the first series half million gallon tanks. It should be a good baseline to use in developing most tanks in the Hanford tank farm.

2. The first data file that is required is a list of risers that are available in the tank. It should be named 'risers' and should be located in the 'data' <tank_name> (i.e. 'data/t106') directory under the main directory structure. The directory name must match the tank name exactly. This is a simple ASCII text file, with one riser name per line. This list should match the names of the riser parts exactly, and should be well known names so that the operator will be able to pick the name from a menu.

3. The second thing that the developer must add is a collision file for each riser. This collision file contains a list of all the tank parts to be included in the collision queue when the LDUA is deployed down that riser. Each collision file must be named exactly the same as the cooresponding riser part listed in the 'risers' file in the 'data' directory. Each collision file must contain a list of all tank parts that are within the reach of the LDUA so that they will be included in the collision queue. Each entry must contain one line for each part to be added to the collision queue, and each part name must be preceded by 'tank:' (i.e. 'tank:ne' for the 'ne' part in the tank setup).

All parts in the tank model could be included in this file, but the preferred method is to reduce the number of polygons in the collision queue by including only those parts which are absolutly necessary. Another method to reduce the size of the queue is to break up the tank itself into multiple parts, some of which will be entirely out of the range of the LDUA and, therefore, will not be included in the collision queue. 
4. Next, the developer must add the tank name to the 'tank.list' file in the 'data' directory. This is done by simply adding a line to the text file with the name of the tank, exactly as it appears in the 'devices/tanks' directory.

5. The developer must minimize the number of polygons that are in the tank model so that the computer is able to render the world fast enough to be useful. However, the developer still must create a model that is accurate enough to satisfy the software requirements.

\subsubsection{Creating New In Tank Hardware Models}

The Add ITH button in the world model software is used to include both the In Tank Hardware that is present in the tank, as well as any devices that may be inserted to monitor the LDUA. Items which are not in range of the LDUA may not be wanted in the tank setup since they add to the load on the computer. As in the case of adding tanks, there is a are a few steps that need to be taken to add ITH to the world model software

1. The first step is to build a device that represents the geometry of the ITH in IGRIP. This model should be saved as a device, in the 'devices/ITH' directory in the main directory structure. Note: it should be saved in a configuration that is representative of a deployment, so that it will be in a deployed state when it is included in the collision queue.

2. Once the device is created, the developer must add the name of the device to the ith.list located in the 'data' directory under the main directory structure. This simply involves adding a line of text with a carriage return at the end of the list.

\subsubsection{Creating New End Effector Models}

New end effectors for the LDUA may be added to the World model software to be more representative of the device than the generic end effector. The process is similar to adding In Tank Hardware to the world model software.

1. The first step is to build a device that represents the geometry of the end effector in IGRIP. This model should be saved as a device, in the 'devices/end_effectors' directory in the main directory structure.

2. Once the device is created, the developer must add the name of the device to the end_effector.list located in the 'data' directory under the main directory structure. This simply involves adding a line of text with a carriage return at the end of the list. 
HNF-SD-TD-OMM-001, Rev. 1

Page 70

\subsubsection{World Model Files and Directories}

\subsubsection{World Model Directory Structure}

All-directories for the world model software are located in the 'WorldModel' subdirectory in the home directory of the oper user. The following is summary of the files that are located in each directory. Specific details of each file are located in the 'Files' section of this document.

'WorldModel/parts' - All geometric part files that make up the three dimensional world model for all tanks, in tank hardware, the Light Duty Utility Arm, and any other item that may be modeled. All parts that are developed for any item to be put into the world model is stored in subdirectories under this directory.

'WorldModel/devices' - IGRIP device files are stored under this directory. This includes in tank hardware, the LDUA, and tank models.

'WorldModel/workcells' - This directory contains the main tank setup for the world model. This tank setup includes only the LDUA. The user buttons will bring all other equipment into the model.

'WorldModel/macros' - This directory contains the macros that correspond to all buttons that make up the user page in IGRIP.

'WorldModel/data' - This directory contains data files that are used for various purposes in the world model software.

'WorldModel/data/'<tank_name $>$ - Each tank will have a directory in the data subdirectory that contains a number of data files specific to that tank.

\subsubsection{World Model Macro Files}

These files contain GSL macros which implement the functions of the user buttons:

choose_tank.gsl - This macro first checks a data file to determine valid tanks that have been developed for the world model software, then loads the tank model into the tank setup.

collision.gsl - This macro turns on and off the near miss check algorithm in IGRIP, and sets the tolerance. The result is that if a part comes within the specified distance from another part in the collision queue, then it highlights the two parts in yellow. If they would collide, the parts turn red.

end_effectors.gsl - This macro loads in specific end effectors into the tank setup, and adds them to the collision queue.

gee.gsl - This macro changes the jaw opening distance on the Gripper End-Effector for display on the World Model. 
ith.gsl - This macro loads in ITH into the tank setup, and loads it into the collision queue, if it is within range.

Ith_remove.gsI - This macro removes ITH from the tank setup

llti_fms.gsl - This macro connects and disconnects the force moment sensor from the World Model

Ilti_real.gsl - This macro connects and disconnects the real robot in the World Model to the physical robot as it is deployed.

Ilti_sim.gsl - This macro connects and disconnects the SPAR hardware simulated robot from the World Model Software.

load.gsl - This macro loads previously saved configurations of deployments.

locate_LDUA.gsl - This macro moves the LDUA into position over a riser by querying the user for which riser, height from the top of the riser to the bottom of the LDUA, and azimuth, yaw, and pitch angles.

postion.gsl - This macro retrieves the position of the LDUA with respect to the World Model Origin.

restart.gsl - This macro returns the software to a previous situation which has been saved in a. data file. This allows the user to restart work quickly after a malfunction any number of parts of the software.

save.gsl - This macro saves data to a file that can be retrieved by the "Load World" button in the World Model Software.

visibility.gsl - This macro makes the LDUA and Simulated LDUA visible or invisible when needed.

\subsubsection{World Model Data Files}

The world model software uses a number of data files that must be formatted correctly for the software to function properly. These files are all located in the $/$ WorldModel/data directory and its subdirectories. All files are simply ASCII character lists of data where each line of each file contains a data string, followed by a carriage return. All files can be edited with simple editors, such as 'vi' or 'jot' on the SGI.

data.world - This file contains all choices made throughout the process of initializing the software. This file is created and read by the software, and should not be edited by the user. It will be overwritten each time the software is started unless the restart button is the first button chosen by the user. The following is an example data.world file. Each line is followed by a description of what the data means. 
HNF-SD-TD-OMM-001, Rev. 1

Page 72

$\begin{array}{ll}\text { ctf } & \text {-- tank name } \\ 12 \text { in } & \text {-- riser for LDUA } \\ 0 & - \text { - yaw angle for LDUA } \\ 0 & \text {-- pitch angle for LDUA } \\ 197 & \text {-- azimuth angle for LDUA } \\ 100 & \text { - height from top of riser to bottom of LDUA } \\ \text { oas } & \text {-- end effector name } \\ \text { tms } & - \text { - 1st in tank hardware (ITH) name } \\ \text { se } & -- \text { 1st in tank hardware riser } \\ \text { generic } & - \text { - 2nd in tank hardware name } \\ 4 & - \text { - for generic ITH specify diameter of ITH (in.) } \\ 200 & - \text { - for generic ITH specify length of ITH (in.) } \\ \text { nw } & - \text { - 2nd in tank hardware riser }\end{array}$

end_effector list - This file contains a list of all end effectors that have been modeled in the software. If a particular end effector hasn't been incorporated into the software yet, the generic option will bring up an end effector that is the maximum size allowable for an end effector. The following is a sample end effector list. Each line contains the name of a valid end effector.

Note, each entry must match the name of a corresponding device in the

'WorldModel/devices/end_effectors' directory.

generic
oas
hrsvs
ssps
nde

ith.list - This file contains a list of the in tank hardware devices that have been modeled in the software. A generic option is available here also, and will establish a cylinder that is a selected diameter and length. The following is a sample ith.list file. Note, each name must match the name of a corresponding device in the 'WorldModel/devices/ITH' directory.

generic

ovs

osvs

tms

Idua.list - This is a list of all parts of the LDUA that are to be included in the collision avoidance checks. The following is a sample ldua.list file. Note, each entry must match the corresponding part in the IGRIP tank setup as: 'LDUA:'<part_name>.

ldua:Idua. 8 
Idua:Idua.7

ldua:Idua. 6

ldua:Idua.5

Idua:Idua. 4

ldua:Idua.3

tank.list - This is a list of all tanks that have been modeled for the software. The following is a sample 'tank.list' file. Note, each entry must match the name of the corresponding tank in the 'WorldModel/devices/tanks' directory.
ctf
$\mathrm{t} 106$
t109

risers - This file is located in the 'WorldModel/data/" $<$ tank_name> directory. It contains a list of all available risers that the LDUA could enter through in the particular tank. The following is a sample risers file. Note, each entry in this file must match the name of a riser in the tank device.
r2
r3
r6
r7
r13

$<$ riser_name $>$ - This file is also located in the 'WorldModel/data/' $<$ tank_name $>$ directory. Each riser listed in the 'risers' file must have a it's own file that lists all the parts that are to be included in the collision queue if the LDUA is deployed through that riser. This is a list of all the parts that are within a $20^{\prime}$ radius of the center of the riser.

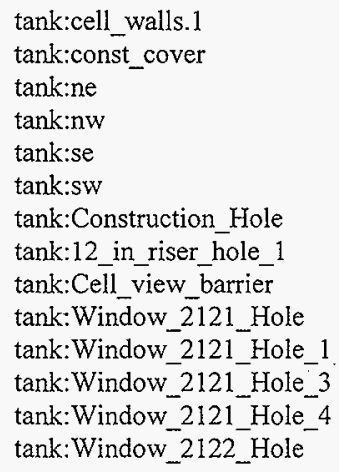


Page 74

\subsection{RECOMMENDED MAINTENANCE}

There is no regular recommended maintenance required for SDAS, other than what is normal for UNIX systems. Regular UNIX system maintenance should be performed by a qualified UNIX systems administrator. Backup should only be required when there are changes to the configuration, or when data is stored on the hard disk. The system as currently configured does not store data on the hard disk.

However, both hardware and software maintenance contracts are recommended. Hanford has site standard maintenance contracts. Participation in these maintenance contract pools allows you to get someone out to repair the equipment with a minimum of fuss and without the delays of bidding a maintenance contract after the equipment failure. 